
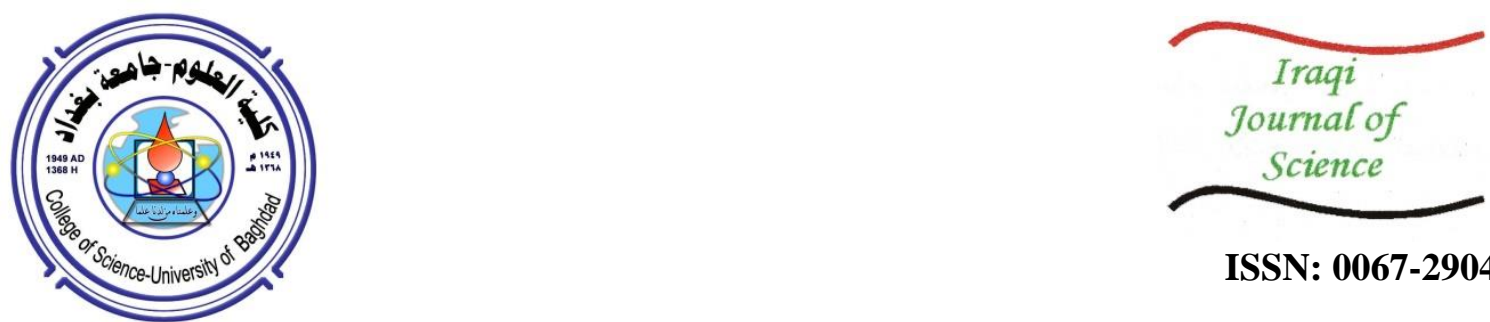

ISSN: 0067-2904

\title{
Asymptotic Stability for Some Types of Nonlinear Fractional Order Differential-Algebraic Control Systems
}

\author{
Sameer Qasim Hasan \\ Department of Mathematics, College of Education, University of Mustansiriyah, Baghdad, Iraq \\ Received: 28/5/2020 \\ Accepted: $30 / 6 / 2020$

\begin{abstract}
The aim of this paper is to study the asymptotically stable solution of nonlinear single and multi fractional differential-algebraic control systems, involving feedback control inputs, by an effective approach that depends on necessary and sufficient conditions.
\end{abstract}

Keywords: Asymptotically Stable, Feedback Control, Differential-Algebraic Equation, Fractional Order, Gronwall inequality, Mult-Fractional Order.

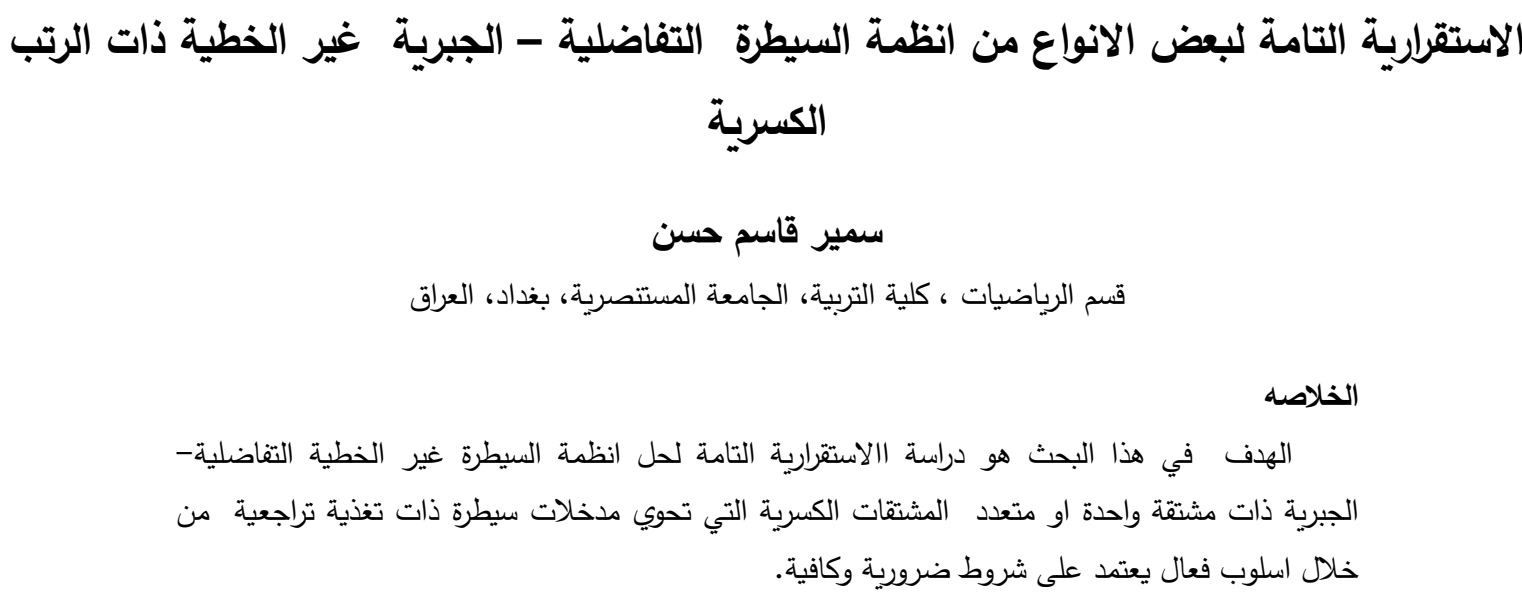

\section{Introduction}

The nonlinear fractional order differential-algebraic control systems appear in a variety of theories and applications. The theory of fractional descriptor ordinary and fractional partial differential equations, with different types of derivatives, have recently been addressed by several researchers for different problems. It is well known that descriptor systems or differential-algebraic systems are the major research fields of the control theory. During the past two decades, differential-algebraic systems attracted much attention due to the comprehensive applications in economics singular systems, not only those containing differential or difference equations as normal systems but also algebraic equations. Thus, their description is considered as being more general. Their class of systems has been widely studied, not only because of theoretical interest but also because of its extensive applications in areas such as robotics and power systems. The necessary and sufficient conditions for the solvability, positivity, and asymptotic stability and stabilization of the fractional descriptor linear systems were established [1-7, 8, 9]. Earlier works $[10,11]$ studied the partial eigenvalue assignment for stabilization of descriptor fractional discrete-time linear systems or by derivative state feedback. In other investigations $[12,10]$, the stabilization problem of singular fractional-order systems with

*Email: dr.sameerqasim@uomustansiriyah.edu.iq 
fractional commensurate fractional order, via static output feedback, was studied . The stability problem of descriptor second-order systems was also considered [13]. Lyapunov equations for stability of second-order systems were established by using Lyapunov method. The robust admissibility problem in singular fractional-order continuous time systems was also studied with a static output feedback controller that is designed for the uncertain closed-loop system to be admissible [14]. Other articles studied the robust stability and stabilization of uncertain fractional-order differential-algebraic nonlinear systems [15, 13].

Our intersect in this paper is to study the asymptotic stability of nonlinear fractional order differential- algebraic control systems, involving feedback input controls. Also, we aim to study single-fractional (15-16) and multi-fractional (21-22) order differential - algebraic control equations .

The following definitions and results are needed later on.

Definition (1.1), [16]

Let $f$ be a function such that $f:[0, \infty) \rightarrow R$. The $\alpha$ fractional order Caputo derivative is defined as $\left({ }_{0}^{c} D_{t}^{\alpha}\right) f(t)=\frac{1}{\Gamma(n-\alpha)} \int_{0}^{t}(t-s)^{n-\alpha-1} f^{(n)}(s) d s, \quad n-1<\alpha<n$, where $\Gamma$ denotes the gamma function.

\section{Lemma (1.1), [17]}

The degree polynomial $a_{n} \lambda^{n}+a_{n-1} \lambda^{n-1}+\cdots+a_{0}=0$ is asymptotically stable if it holds the following condition: $\left|\arg \lambda_{i}\right|>\alpha \frac{\pi}{2}$ for all zeros $\lambda_{i}, i=1, \ldots, n$.

\section{Definition (1.1), [18]}

The following fractional order system

$$
\left({ }_{0}^{c} D_{t}^{\alpha}\right) x(t)=A x(t)
$$

$x(0)=x_{0}, 0<\alpha<1$,

where $\mathrm{x}=\left(x_{1}, x_{2}, \ldots, x_{n}\right)^{T}$ and $x_{0}=\left(x_{10}, x_{20}, \ldots, x_{n 0}\right)^{T}, A \in R^{n \times n}$ is

a) stable if for any $x_{0}$ there exists $\epsilon>0$ such that $\|x\| \leq \epsilon$ for $t \geq 0$.

b) asymptotically stable if $\lim _{t \rightarrow \infty}\|x\|=0$.

\section{Lemma (1.2), [18]}

Consider the linear fractional control system

$$
\left({ }_{0}^{c} D_{t}^{\alpha}\right) x(t)=A x(t)+B u(t)
$$

$x(0)=x_{0}, 0<\alpha \leq 1$, where $\mathrm{x}=\left(x_{1}, x_{2}, \ldots, x_{n}\right)^{T}$ and $x_{0}=\left(x_{10}, x_{20}, \ldots, x_{n 0}\right)^{T}$,

$u \in L\left([0, \infty], R^{m}\right), A \in R^{n \times n} B \in R^{n \times m}$. The system is stable if and only if $\left|\arg \lambda_{i}\right|>\alpha \frac{\pi}{2}$,

for all zeros $\lambda_{i}, i=1, \ldots, n$.

\section{Nonlinear Fractional Order Differential-Algebraic Control Systems}

The following two types of nonlinear fractional order differential-algebraic control systems are presented.

\subsection{Single- Fractional Order Differential -Algebraic Control Equations}

Consider the nonlinear single fractional order differential -algebraic control system:

$$
\begin{gathered}
\left({ }_{0}^{c} D_{t}^{\alpha}\right) x_{1}(t)=\sum_{i=1}^{3} a_{1 i} x_{i}(t)+\sum_{j=1}^{2} b_{1 j} u_{j}(t)+f_{1}\left(x_{1}, x_{2}, x_{3}\right) g_{1}\left({ }_{0}^{c} D_{t}^{\beta} x_{1},{ }_{0}^{c} D_{t}^{\beta} x_{2},{ }_{0}^{c} D_{t}^{\beta} x_{3}\right) \\
\left({ }_{0}^{c} D_{t}^{\alpha}\right) x_{2}(t)=\sum_{i=1}^{3} a_{2 i} x_{i}(t)+\sum_{j=1}^{2} b_{2 j} u_{j}(t)+f_{2}\left(x_{1}, x_{2}, x_{3}\right) g_{2}\left({ }_{0}^{c} D_{t}^{\gamma} x_{1},{ }_{0}^{c} D_{t}^{\gamma} x_{2},{ }_{0}^{c} D_{t}^{\gamma} x_{3}\right) \\
x_{3}(t)=\sum_{i=1}^{3} a_{3 i} x_{i}(t)+\sum_{j=1}^{2} b_{3, j} u_{j}(t)+f_{3}\left(x_{1}, x_{2}, x_{3}\right) g_{3}\left({ }_{0}^{c} D_{t}^{\delta} x_{1},{ }_{0} D_{t}^{\delta} x_{2},{ }_{0}^{c} D_{t}^{\delta} x_{3}\right)
\end{gathered}
$$

where $a_{1 i}, a_{2 i}, a_{3 i}, b_{1}, b_{2}$, and $b_{3}$ are constants, $x_{i} \in R$ are state vectors, $\mathrm{i}=1 \ldots 3,0 \leq \alpha, \beta, \gamma, \delta \leq 1$ $u_{j}(t) \in R, j=1,2$ are control input functions, and $f_{i}, \mathrm{~g}_{i}, \mathrm{i}=1 \ldots 3$ are varying nonlinear time values . The linear system of (1-3) is:

$$
\begin{gathered}
\left({ }_{0}^{c} D_{t}^{\alpha}\right) x_{1}(t)=\sum_{i=1}^{3} a_{1 i} x_{i}(t)+\sum_{j=1}^{2} b_{1, j} u_{j}(t) \\
\left({ }_{0}^{c} D_{t}^{\alpha}\right) x_{2}(t)=\sum_{i=1}^{3} a_{2 i} x_{i}(t)+\sum_{j=1}^{2} b_{2, j} u_{j}(t) \\
x_{3}(t)=\sum_{i=1}^{3} a_{3 i} x_{i}(t)+\sum_{i=1}^{2} b_{3, i} u_{i}(t)
\end{gathered}
$$

From (6), it is given that :

Hence,

$$
x_{3}(t)=\frac{a_{31} x_{1}(t)}{1-a_{33}}+\frac{a_{32} x_{2}(t)}{1-a_{33}}+\frac{\sum_{i=1}^{2} b_{3, i} u_{i}(t)}{1-a_{33}}
$$

$$
\left({ }_{0}^{c} D_{t}^{\alpha}\right) x_{1}(t)=a_{11} x_{1}(t)+a_{12} x_{2}(t)+a_{13}\left[\frac{a_{31} x_{1}(t)}{1-a_{33}}+\frac{a_{32} x_{2}(t)}{1-a_{33}}+\frac{\sum_{i=1}^{2} b_{3, i} u_{i}(t)}{1-a_{33}}\right]+\sum_{j=1}^{2} b_{1 j} u_{j}(t)
$$


$\left({ }_{0}^{c} D_{t}^{\alpha}\right) x_{2}(t)=a_{21} x_{1}(t)+a_{22} x_{2}(t)+a_{23}\left[\frac{a_{31} x_{1}(t)}{1-a_{33}}+\frac{a_{32} x_{2}(t)}{1-a_{33}}+\frac{\sum_{i=1}^{2} b_{3, i} u_{i}(t)}{1-a_{33}}\right]+\sum_{j=1}^{2} b_{2 j} u_{j}(t)$ then,

$$
\begin{aligned}
& \left({ }_{0}^{c} D_{t}^{\alpha}\right) x_{1}(t)=\left[a_{11}+\frac{a_{13} a_{31}}{1-a_{33}}\right] x_{1}(t)+\left[a_{12}+\frac{a_{13} a_{32}}{1-a_{33}}\right] x_{2}(t)+\left[a_{13} \frac{\sum_{i=1}^{2} b_{3, i} u_{i}(t)}{1-a_{33}}\right]+\sum_{i=1}^{2} b_{1 i} u_{i}(t) \\
& \left({ }_{0}^{c} D_{t}^{\alpha}\right) x_{2}(t)=\left[a_{21}+\frac{a_{23} a_{31}}{1-a_{33}}\right] x_{1}(t)+\left[a_{22}+\frac{a_{23} a_{32}}{1-a_{33}}\right] x_{2}(t)+\left[a_{23} \frac{\sum_{i=1}^{2} b_{3, i} u_{i}(t)}{1-a_{33}}\right]+\sum_{i=1}^{2} b_{2, i} u_{i}(t)
\end{aligned}
$$

For more simplicity, let $\tilde{a}_{11}=a_{11}+\frac{a_{13} a_{31}}{1-a_{33}} \quad, \tilde{a}_{12}=a_{12}+\frac{a_{13} a_{32}}{1-a_{33}} \quad, \tilde{a}_{21}=a_{21}+\frac{a_{23} a_{31}}{1-a_{33}}$

$$
\begin{aligned}
& , \tilde{a}_{22}=a_{22}+\frac{a_{23} a_{32}}{1-a_{33}}, \tilde{a}_{31}=\frac{a_{31}}{1-a_{33}} \quad, \tilde{a}_{32}=\frac{a_{32}}{1-a_{33}}, \tilde{b}_{11}=\frac{a_{13} b_{3,1}}{1-a_{33}}+b_{11}, \tilde{b}_{12}=\frac{a_{13} b_{3,2}}{1-a_{33}}+b_{12}, \\
& \tilde{b}_{21}=\frac{a_{23} b_{31}}{1-a_{33}}+b_{21}, \tilde{b}_{22}=\frac{a_{23} b_{32}}{1-a_{33}}+b_{22}, \tilde{b}_{31}=\frac{b_{31}}{1-a_{33}} \sum_{i=0}^{T} a_{33}^{i}, \tilde{b}_{32}=\frac{b_{32}}{1-a_{33}} \sum_{i=0}^{T} a_{33}^{i}
\end{aligned}
$$

Equations (7) and (8) become,

$$
\begin{gathered}
\left({ }_{0}^{c} D_{t}^{\alpha}\right) x_{1}(t)=\tilde{a}_{11} x_{1}(t)+\tilde{a}_{12} x_{2}(t)+\sum_{j=1}^{2} \tilde{b}_{1 j} u_{j}(t) \\
\left({ }_{0}^{c} D_{t}^{\alpha}\right) x_{2}(t)=\tilde{a}_{21} x_{1}(t)+\tilde{a}_{22} x_{2}(t)+\sum_{j=1}^{2} \tilde{b}_{2 j} u_{j}(t) \\
x_{3}(t)=\tilde{a}_{31} x_{1}(t)+\tilde{a}_{32} x_{2}(t)+\sum_{j=1}^{2} \tilde{b}_{3 j} u_{j}(t)
\end{gathered}
$$

Now, we consider the following related linear feedback control system

$$
\begin{aligned}
& \left({ }_{0}^{c} D_{t}^{\alpha_{1}}\right) x_{1 \rho}(t)+\left({ }_{0}^{c} D_{t}^{\alpha_{2}}\right) x_{1 \rho}(t)=\tilde{a}_{11 \rho} x_{1 \rho}(t)+\tilde{a}_{12 \rho} x_{2 \rho}(t)+\tilde{b}_{11 \rho} x_{3}(t)+\tilde{b}_{12 \rho} x_{3}(t) \\
& \left({ }_{0}^{c} D_{t}^{\alpha_{1}}\right) x_{2 \rho}(t)+\left({ }_{0}^{c} D_{t}^{\alpha_{2}}\right) x_{2 \rho}(t)=\tilde{a}_{21 \rho} x_{1 \rho}(t)+\tilde{a}_{22 \rho} x_{2 \rho}(t)+\tilde{b}_{21 \rho} x_{3}(t)+\tilde{b}_{22 \rho} x_{3}(t) \\
& u_{1}(t)=K_{\rho} x_{1 \rho}(t) u_{2}(t)=K_{\rho} x_{2 \rho}(t)
\end{aligned}
$$

where $x_{1 \rho}, x_{2 \rho} \in R, \tilde{a}_{11 \rho}, \tilde{a}_{12 \rho}, \tilde{a}_{21 \rho}, \tilde{a}_{22 \rho}$, and $K_{\rho}$ are constants.

For the nonlinear multi-fractional order differential -algebraic control system in equations (10-11) with equations (12-13), we obtain:

$$
\left[\begin{array}{l}
\left({ }_{0}^{c} D_{t}^{\alpha}\right) x_{1}(t) \\
\left(\begin{array}{c}
c \\
0
\end{array} D_{t}^{\alpha}\right) x_{2}(t) \\
\left({ }_{0}^{c} D_{t}^{\alpha}\right) x_{1, \rho}(t) \\
\left({ }_{0}^{c} D_{t}^{\alpha}\right) x_{2, \rho}(t)
\end{array}\right]
$$

$=$

$$
\begin{aligned}
& {\left[\begin{array}{cccc}
\tilde{a}_{11} & \tilde{a}_{12} & \tilde{b}_{11} K_{\rho} & \tilde{b}_{12} K_{\rho} \\
\tilde{a}_{21} & \tilde{a}_{22} & \tilde{b}_{2,1} K_{\rho} & \tilde{b}_{2,2} K_{\rho} \\
\tilde{a}_{31}\left(\tilde{b}_{11 \rho}+\tilde{b}_{12 \rho}\right) & \tilde{a}_{32}\left(\tilde{b}_{11 \rho}+\tilde{b}_{12 \rho}\right) & \sum_{j=1}^{2} \tilde{b}_{1 j \rho} \tilde{b}_{31} K_{\rho}+\tilde{a}_{11 \rho} & \sum_{j=1}^{2} \tilde{b}_{1 j \rho} \tilde{b}_{32} K_{\rho}+\tilde{a}_{12 \rho} \\
\tilde{a}_{31}\left(\tilde{b}_{21 \rho}+\tilde{b}_{22 \rho}\right) & \tilde{a}_{32}\left(\tilde{b}_{21 \rho}+\tilde{b}_{22 \rho}\right) & \sum_{j=1}^{2} \tilde{b}_{2 j \rho} \tilde{b}_{31} K_{\rho}+\tilde{a}_{21 \rho} & \sum_{j=1}^{2} \tilde{b}_{2 j \rho} \tilde{b}_{32} K_{\rho}+\tilde{a}_{22 \rho}
\end{array}\right]\left[\begin{array}{c}
x_{1}(t) \\
x_{2}(t) \\
x_{1, \rho}(t) \\
x_{2, \rho}(t)
\end{array}\right]} \\
& +\left[\begin{array}{c}
f_{1}\left(x_{1}, x_{2}, x_{3}\right) \mathrm{g}_{1}\left({ }_{0}^{c} D_{t}^{\beta} x_{1},{ }_{0}^{c} D_{t}^{\beta} x_{2},{ }_{0}^{c} D_{t}^{\beta} x_{3}\right) \\
f_{2}\left(x_{1}, x_{2}, x_{3}\right) g_{2}\left({ }_{0}^{c} D_{t}^{\beta} x_{1},{ }_{0}^{c} D_{t}^{\beta} x_{2},{ }_{0}^{c} D_{t}^{\beta} x_{3}\right) \\
\tilde{b}_{1,2, \rho} f_{3}\left(x_{1}, x_{2}, x_{3}\right) g_{3}\left({ }_{0}^{c} D_{t}^{\beta} x_{1},{ }_{0}^{c} D_{t}^{\beta} x_{2},{ }_{0}^{c} D_{t}^{\beta} x_{3}\right) \\
\tilde{b}_{2,2, \rho} f_{3}\left(x_{1}, x_{2}, x_{3}\right) g_{3}\left({ }_{0}^{c} D_{t}^{\beta} x_{1},{ }_{0}^{c} D_{t}^{\beta} x_{2},{ }_{0}^{c} D_{t}^{\beta} x_{3}\right)
\end{array}\right] \\
& x_{3}(t)=\left[\begin{array}{llll}
\tilde{a}_{31} & \tilde{a}_{32} & \tilde{b}_{3,1} K_{\rho} & \tilde{b}_{3,2} K_{\rho}
\end{array}\right]\left[\begin{array}{c}
x_{1}(t) \\
x_{2}(t) \\
x_{1, \rho}(t) \\
x_{2, \rho}(t)
\end{array}\right]+f_{3}\left(x_{1}, x_{2}, x_{3}\right) g_{3}\left({ }_{0}^{c} D_{t}^{\beta} x_{1},{ }_{0}^{c} D_{t}^{\beta} x_{2},{ }_{0}^{c} D_{t}^{\beta} x_{3}\right)
\end{aligned}
$$

Thus,

$$
\begin{aligned}
& \left({ }_{0}^{c} D_{t}^{\alpha}\right) x(t)=A x+F(x) G\left({ }_{0}^{c} D_{t}^{\alpha} x\right) \\
& x_{3}(t)=B x+f_{3}(x) g_{3}\left({ }_{0}^{c} D_{t}^{\alpha} x\right) \\
& \text { where }
\end{aligned}
$$

$$
A=\left[\begin{array}{cccc}
\tilde{a}_{11} & \tilde{a}_{12} & \tilde{b}_{11} K_{\rho} & \tilde{b}_{12} K_{\rho} \\
\tilde{a}_{21} & \tilde{a}_{22} & \tilde{b}_{2,1} K_{\rho} & \tilde{b}_{2,2} K_{\rho} \\
\tilde{a}_{31}\left(\tilde{b}_{11 \rho}+\tilde{b}_{12 \rho}\right) & \tilde{a}_{32}\left(\tilde{b}_{11 \rho}+\tilde{b}_{12 \rho}\right) & \sum_{j=1}^{2} \tilde{b}_{1 j \rho} \tilde{b}_{31} K_{\rho}+\tilde{a}_{11 \rho} & \sum_{j=1}^{2} \tilde{b}_{1 j \rho} \tilde{b}_{32} K_{\rho}+\tilde{a}_{12 \rho} \\
\tilde{a}_{31}\left(\tilde{b}_{21 \rho}+\tilde{b}_{22 \rho}\right) & \tilde{a}_{32}\left(\tilde{b}_{21 \rho}+\tilde{b}_{22 \rho}\right) & \sum_{j=1}^{2} \tilde{b}_{2 j \rho} \tilde{b}_{31} K_{\rho}+\tilde{a}_{21 \rho} & \sum_{j=1}^{2} \tilde{b}_{2 j \rho} \tilde{b}_{32} K_{\rho}+\tilde{a}_{22 \rho}
\end{array}\right]
$$


$B=\left[\begin{array}{llll}\tilde{a}_{31} & \tilde{a}_{32} & \tilde{b}_{31} K_{\rho} & \tilde{b}_{32} K_{\rho}\end{array}\right], F(x) G\left({ }_{0}^{c} D_{t}^{\alpha} x\right)=\left[\begin{array}{c}f_{1}\left(x_{1}, x_{2}, x_{3}\right) \mathrm{g}_{1}\left({ }_{0}^{c} D_{t}^{\beta} x_{1},{ }_{0}^{c} D_{t}^{\beta} x_{2},{ }_{0}^{c} D_{t}^{\beta} x_{3}\right) \\ f_{2}\left(x_{1}, x_{2}, x_{3}\right) g_{2}\left({ }_{0}^{c} D_{t}^{\beta} x_{1},{ }_{0}^{c} D_{t}^{\beta} x_{2},{ }_{0}^{c} D_{t}^{\beta} x_{3}\right) \\ \tilde{b}_{1,2, \rho} f_{3}\left(x_{1}, x_{2}, x_{3}\right) g_{3}\left({ }_{0}^{c} D_{t}^{\beta} x_{1},{ }_{0}^{c} D_{t}^{\beta} x_{2},{ }_{0}^{c} D_{t}^{\beta} x_{3}\right) \\ \tilde{b}_{2,2, \rho} f_{3}\left(x_{1}, x_{2}, x_{3}\right) g_{3}\left({ }_{0}^{c} D_{t}^{\beta} x_{1},{ }_{0}^{c} D_{t}^{\beta} x_{2},{ }_{0}^{c} D_{t}^{\beta} x_{3}\right)\end{array}\right]$ $f_{3}(x) g_{3}\left({ }_{0}^{c} D_{t}^{\alpha} x\right)=f_{3}\left(x_{1}, x_{2}, x_{3}\right) g_{3}\left({ }_{0}^{c} D_{t}^{\beta} x_{1},{ }_{0}^{c} D_{t}^{\beta} x_{2},{ }_{0}^{c} D_{t}^{\beta} x_{3}\right)$.

\section{Lemma (2.1.1), [19]}

The Mittage-Leffler function $E_{\alpha, \beta}\left(A t^{\alpha}\right)$ satisfies the following

i. $\quad \mathrm{E}_{\alpha, 1}\left(\mathrm{At}^{\alpha}\right) \leq \mathrm{K}_{\mathrm{E}_{\alpha, 1}}\left\|\mathrm{e}^{A \mathrm{t}^{\alpha}}\right\|, \alpha>1$.

ii. $\mathrm{E}_{\alpha, \alpha}\left(\mathrm{At}^{\alpha}\right) \leq \mathrm{K}_{\mathrm{E}_{\alpha, \alpha}}\left\|\mathrm{e}^{\mathrm{At}{ }^{\alpha}}\right\|, \alpha>1$,

where $\mathrm{A} \in R^{n x n}, \mathrm{~K}_{\mathrm{E}_{\alpha, 1}} \mathrm{~K}_{\mathrm{E}_{\alpha, \alpha}}$ are finite real constants such that $\mathrm{K}_{\mathrm{E}_{\alpha, 1}}>1, \mathrm{~K}_{\mathrm{E}_{\alpha, \alpha}}>1$.

\section{Lemma (2.1.2), [19]}

Let $\alpha>0 \mathrm{v}(\mathrm{t})$ be a nonnegative function that is locally integrable on $[0, \mathrm{~T})$, let a(t) be a nonnegative,nondecreasing continuous function that is defined on $[0, T)$, and let $a(t)<M$. Suppose that $z(t)$ is nonnegative and locally integrable on $[0, T)$ with $z(t) \leq v(t)+a(t) \int_{0}^{t}(t-\tau)^{\alpha-1} z(\tau) d \tau$. If $\mathrm{v}(t)$ is a non-decreasing function on $[0, \mathrm{~T})$, then we have

$\mathrm{z}(\mathrm{t}) \leq \mathrm{v}(\mathrm{t}) \mathrm{E}_{\alpha}\left(\Gamma(\alpha) \mathrm{a}(\mathrm{t}) \mathrm{t}^{\alpha}\right)$.

\section{Theorem (2.1.3)}

Suppose that the following nonlinear fractional order differential-algebraic control system (17-18) with feedback control (14) satisfies the following conditions:

1. $\quad \operatorname{Re}(\operatorname{eig}(\mathrm{A}))<0 \quad$ and $\quad-\max \operatorname{Re}(\operatorname{eig}(\mathrm{A}))>\Gamma(\alpha)$

2. $\left\|F(x) G\left({ }_{0}^{c} D_{t}^{\alpha} x\right)\right\|=\left\|f_{3}(x)\right\|=o(\|x\|)$ as $\|x\| \rightarrow 0$.

where $F(x)=\left[\begin{array}{c}f_{1}\left(x_{1}, x_{2}, x_{3}\right) g_{1}\left({ }_{0}^{c} D_{t}^{\beta} x_{1},{ }_{0}^{c} D_{t}^{\beta} x_{2},{ }_{0}^{c} D_{t}^{\beta} x_{3}\right) \\ f_{2}\left(x_{1}, x_{2}, x_{3}\right) g_{2}\left({ }_{0}^{c} D_{t}^{\beta} x_{1},{ }_{0}^{c} D_{t}^{\beta} x_{2},{ }_{0}^{c} D_{t}^{\beta} x_{3}\right) \\ \tilde{b}_{2,2, \rho} f_{3}\left(x_{1}, x_{2}, x_{3}\right) \mathrm{g}_{3}\left({ }_{0}^{c} D_{t}^{\beta} x_{1},{ }_{0}^{c} D_{t}^{\beta} x_{2},{ }_{0}^{c} D_{t}^{\beta} x_{3}\right) \\ \tilde{b}_{1,2, \rho} f_{3}\left(x_{1}, x_{2}, x_{3}\right) \mathrm{g}_{3}\left({ }_{0}^{c} D_{t}^{\beta} x_{1},{ }_{0}^{c} D_{t}^{\beta} x_{2},{ }_{0}^{c} D_{t}^{\beta} x_{3}\right)\end{array}\right]$ and $K_{g_{i}}, \mathrm{i}=1, . .3$ are the nonnegative

continuous function, and $\mu_{g_{i}}$ are the continuous non-decreasing positive functions, such that $\left\|g_{i}\left(t,{ }_{0}^{c} D_{t}^{\alpha} x\right)\right\| \leq K_{g_{i}}(t) \mu_{g_{i}}\left(\left\|_{0}^{c} D_{t}^{\alpha} x\right\|\right), \mathrm{i}=1, . .3$.

Then, the system (15) is a locally asymptotically stable.

\section{Proof}

By taking the Laplace transformation to (17), we get

$s^{\alpha} X(s)-s^{\alpha-1} x_{0}=\tilde{A} X(s)+\mathcal{L}\left\{F(x) G\left({ }_{0}^{c} D_{t}^{\beta}\right)\right\}$, thus

$$
X(s)=\left(I s^{\alpha}-\tilde{A}\right)^{-1}\left(-s^{\alpha-1} x_{0}+\mathcal{L}\left\{F(x) G\left({ }_{0}^{c} D_{t}^{\beta} x\right)\right\}\right)
$$

By taking the Laplace inverse transformation to (19), we obtain

$\mathrm{x}(\mathrm{t})=E_{\alpha, 1}\left(\tilde{A} t^{\alpha}\right) x_{0}+\int_{0}^{t}(t-\tau)^{\alpha-1} E_{\alpha, \alpha}\left(\tilde{A}(t-\tau)^{\alpha}\right) F(x(\tau)) G\left({ }_{0}^{c} D_{t}^{\beta} x(\tau) \mathrm{d} \tau\right.$

From lemma (2.1.1), we have

$\|x(t)\|=L_{1}\left\|e^{\tilde{A} t^{\alpha}}\right\|\left\|x_{0}\right\|+L_{3} \int_{0}^{t}(t-\tau)^{\alpha-1}\left\|e^{\tilde{A}(t-\tau)^{\alpha}}\right\| \| F(x(\tau)) G\left({ }_{0}^{c} D_{t}^{\beta} x(\tau) \| \mathrm{d} \tau\right.$

From condition (1), the matrix $\tilde{A}$ is stable and there is a constant $L_{4}>0$ such that $\left\|e^{\tilde{A} t^{\alpha}}\right\| \leq L_{4} e^{-\omega t^{\alpha}}$, hence,

$\|x(t)\| \leq L_{1} L_{4} e^{-\omega t^{\alpha}}\left\|x_{0}\right\|+L_{3} L_{4} \int_{0}^{t}(t-\tau)^{\alpha-1} e^{-\omega(t-\tau)^{\alpha}} \| F(x(\tau)) G\left({ }_{0}^{c} D_{t}^{\beta} x(\tau) \| \mathrm{d} \tau\right.$

From condition (2), we get

$$
\begin{aligned}
& \| F(x(\tau)) G\left({ }_{0}^{c} D_{t}^{\beta} x(\tau) \|=\right. \\
& \left\|f_{1}\left(x_{1}, x_{2}, x_{3}\right) g_{1}\left({ }_{0}^{c} D_{t}^{\beta} x_{1},{ }_{0}^{c} D_{t}^{\beta} x_{2},{ }_{0}^{c} D_{t}^{\beta} x_{3}\right)\right\|+\left\|f_{2}\left(x_{1}, x_{2}, x_{3}\right) g_{2}\left({ }_{0}^{c} D_{t}^{\beta} x_{1},{ }_{0}^{c} D_{t}^{\beta} x_{2},{ }_{0}^{c} D_{t}^{\beta} x_{3}\right)\right\|+
\end{aligned}
$$


$\left\|\tilde{b}_{2,2, \rho} f_{3}\left(x_{1}, x_{2}, x_{3}\right) \mathrm{g}_{3}\left({ }_{0}^{c} D_{t}^{\beta} x_{1},{ }_{0}^{c} D_{t}^{\beta} x_{2},{ }_{0}^{c} D_{t}^{\beta} x_{3}\right)\right\|+$

$\left\|\tilde{b}_{1,2, \rho} f_{3}\left(x_{1}, x_{2}, x_{3}\right) g_{3}\left({ }_{0}^{c} D_{t}^{\beta} x_{1},{ }_{0}^{c} D_{t}^{\beta} x_{2},{ }_{0}^{c} D_{t}^{\beta} x_{3}\right)\right\|$

$\leq\left\|f_{1}\left(x_{1}, x_{2}, x_{3}\right)\right\| K_{g_{1}}(t) \mu_{g_{1}}\left(\left\|_{0}^{c} D_{t}^{\alpha} x\right\|\right)+\left\|f_{2}\left(x_{1}, x_{2}, x_{3}\right)\right\| K_{g_{2}}(t) \mu_{g_{2}}\left(\left\|{ }_{0}^{c} D_{t}^{\alpha} x\right\|\right)$

$+\left|\tilde{b}_{2,2, \rho}\right|\left\|f_{3}\left(x_{1}, x_{2}, x_{3}\right)\right\| K_{g_{3}}(t) \mu_{g_{3}}\left(\left\|{ }_{0}^{c} D_{t}^{\alpha} x\right\|\right)+\left|\tilde{b}_{1,2, \rho}\right|\left\|f_{3}\left(x_{1}, x_{2}, x_{3}\right)\right\| K_{g_{3}}(t) \mu_{g_{3}}\left(\left\|{ }_{0}^{c} D_{t}^{\alpha} x\right\|\right)$.

Since $\left\|F(x) G\left({ }_{0}^{c} D_{t}^{\alpha} x\right)\right\|=o(\|x\|)$ as $\|x\| \rightarrow 0$, thus $\lim _{\|x\| \rightarrow 0} \frac{\left\|F(x) G\left({ }_{0}^{c} D_{t}^{\alpha} x\right)\right\|}{\|x\|}=0$, which implies that $\frac{\left\|F(x) G\left({ }_{0}^{c} D_{t}^{\alpha} x\right)\right\|}{\|x\|}<\frac{1}{L_{3} L_{4}}$, therefore

$\| F(x(\tau)) G\left({ }_{0}^{c} D_{t}^{\beta} x(\tau) \| \leq \frac{1}{L_{3} L_{4}}\left[K_{g_{1}}(t) \mu_{g_{1}}\left(\left\|{ }_{0}^{c} D_{t}^{\alpha} x\right\|\right)+\left\|f_{2}\left(x_{1}, x_{2}, x_{3}\right)\right\| K_{g_{2}}(t) \mu_{g_{2}}\left(\left\|{ }_{0}^{c} D_{t}^{\alpha} x\right\|\right)+\right.\right.$

$\left.\left(\left|\tilde{b}_{2,2, \rho}\right|+\left|\tilde{b}_{1,2, \rho}\right|\right) K_{g_{3}}(t) \mu_{g_{3}}\left(\left\|{ }_{0}^{c} D_{t}^{\alpha} x\right\|\right)\right]\|x\|$.

Now,we set that $L_{5}=\left[K_{g_{1}}(t) \mu_{g_{1}}\left(\left\|_{0}^{c} D_{t}^{\alpha} x\right\|\right)+\left\|f_{2}\left(x_{1}, x_{2}, x_{3}\right)\right\| K_{g_{2}}(t) \mu_{g_{2}}\left(\left\|{ }_{0}^{c} D_{t}^{\alpha} x\right\|\right)\right]$

We have

$$
\left.+\left(\left|\tilde{b}_{2,2, \rho}\right|+\left|\tilde{b}_{1,2, \rho}\right|\right) K_{g_{3}}(t) \mu_{g_{3}}\left(\left\|_{0}^{c} D_{t}^{\alpha} x\right\|\right)\right]
$$

$\|x(t)\| \leq L_{1} L_{4} e^{-\omega t^{\alpha}}\left\|x_{0}\right\|+L_{5} \int_{0}^{t}(t-\tau)^{\alpha-1} e^{-\omega(t-\tau)^{\alpha}}\|x(\tau)\| \mathrm{d} \tau$, thus

$e^{\omega t}\|x(t)\| \leq L_{1} L_{4} e^{-\omega t^{\alpha}}\left\|x_{0}\right\|+L_{5} \int_{0}^{t}(t-\tau)^{\alpha-1} e^{\omega \tau}\|x(\tau)\|$

By using Gronwall inequality and lemma(2.1.2), we have

$e^{\omega t}\|x(t)\| \leq L_{1} L_{4}\left\|x_{0}\right\| E_{\alpha, 1}\left(L_{5} \Gamma(\alpha) t^{\alpha}\right) \leq L_{1} L_{4}\left\|x_{0}\right\| e^{L_{5} \Gamma(\alpha) t^{\alpha}}$, then

$\|x(t)\| \leq c_{1} L_{1} L_{4}\left\|x_{0}\right\| e^{-\left(\omega-L_{5} \Gamma(\alpha)\right) t^{\alpha}}$

As $\rightarrow \infty,\|x(t)\| \rightarrow 0$ for $\omega>L_{5} \Gamma(\alpha)^{1 / \alpha}$.

From condition (2), we have that $x \rightarrow 0$ as $t \rightarrow \infty, x_{3}(t)=B x+f_{3}(x) g_{3}\left({ }_{0}^{c} D_{t}^{\alpha} x\right)$. We get

$\left\|x_{3}(t)\right\| \leq\|B x\|+\left\|f_{3}(x)\right\|\left\|g_{3}\left(t,{ }_{0}^{c} D_{t}^{\alpha}(x)\right)\right\|$. Now, since $\left\|f_{3}(x)\right\|=o(\|x\|)$ as $\|x\| \rightarrow 0$, this implies that $\lim _{\|x\| \rightarrow 0} \frac{\left\|f_{3}(x)\right\|}{\|x\|}=0$, so that $\frac{\left\|f_{3}(x)\right\|}{\|x\|}<\delta_{1}$, and since $\left\|g_{3}\left(t,{ }_{0}^{c} D_{t}^{\alpha}(x)\right)\right\| \leq K_{g_{3}}(t)$ $\mu_{g_{3}}\left(\left\|{ }_{0}^{c} D_{t}^{\alpha} x\right\|\right)$, therefore

$\left\|x_{3}(t)\right\| \leq\left(\|B\|+\delta_{1} K_{g_{3}}(t) \mu_{g_{3}}\left(\left\|_{0}^{c} D_{t}^{\alpha} x\right\|\right)\right)\|x\|$.

Hence, $\left\|x_{3}(t)\right\| \rightarrow 0$, which implies that system (17-18) is asymptotically stable.

Example (2.1.2)

The following single-fractional order differential-algebraic equations (19-20) with Caputo derivative are asymptotically stable, as follows

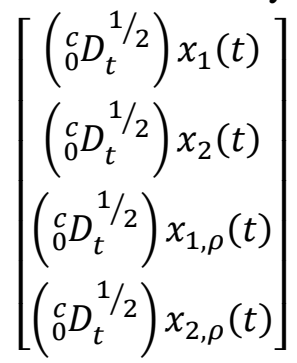

$=$

$\left[\begin{array}{cccc}\tilde{a}_{11} & \tilde{a}_{12} & \tilde{b}_{11} K_{\rho} & \tilde{b}_{12} K_{\rho} \\ \tilde{a}_{21} & \tilde{a}_{22} & \tilde{b}_{2,1} K_{\rho} & \tilde{b}_{2,2} K_{\rho} \\ \tilde{a}_{31}\left(\tilde{b}_{11 \rho}+\tilde{b}_{12 \rho}\right) & \tilde{a}_{32}\left(\tilde{b}_{11 \rho}+\tilde{b}_{12 \rho}\right) & \sum_{j=1}^{2} \tilde{b}_{1 j \rho} \tilde{b}_{31} K_{\rho}+\tilde{a}_{11 \rho} & \sum_{j=1}^{2} \tilde{b}_{1 j \rho} \tilde{b}_{32} K_{\rho}+\tilde{a}_{12 \rho} \\ \tilde{a}_{31}\left(\tilde{b}_{21 \rho}+\tilde{b}_{22 \rho}\right) & \tilde{a}_{32}\left(\tilde{b}_{21 \rho}+\tilde{b}_{22 \rho}\right) & \sum_{j=1}^{2} \tilde{b}_{2 j \rho} \tilde{b}_{31} K_{\rho}+\tilde{a}_{21 \rho} & \sum_{j=1}^{2} \tilde{b}_{2 j \rho} \tilde{b}_{32} K_{\rho}+\tilde{a}_{22 \rho}\end{array}\right]\left[\begin{array}{c}x_{1}(t) \\ x_{2}(t) \\ x_{1 \rho}(t) \\ x_{2 \rho}(t)\end{array}\right]$

$+\left[\begin{array}{c}f_{1}\left(x_{1}, x_{2}, x_{3}\right) g_{1}\left({ }_{0}^{c} D_{t}^{\beta} x_{1},{ }_{0}^{c} D_{t}^{\beta} x_{2},{ }_{0}^{c} D_{t}^{\beta} x_{3}\right) \\ f_{2}\left(x_{1}, x_{2}, x_{3}\right) g_{2}\left({ }_{0}^{c} D_{t}^{\beta} x_{1},{ }_{0}^{c} D_{t}^{\beta} x_{2},{ }_{0}^{c} D_{t}^{\beta} x_{3}\right) \\ \tilde{b}_{12} f_{3}\left(x_{1}, x_{2}, x_{3}\right) g_{3}\left({ }_{0}^{c} D_{t}^{\beta} x_{1},{ }_{0}^{c} D_{t}^{\beta} x_{2},{ }_{0}^{c} D_{t}^{\beta} x_{3}\right) \\ \tilde{b}_{22 \rho} f_{3}\left(x_{1}, x_{2}, x_{3}\right) g_{3}\left({ }_{0}^{c} D_{t}^{\beta} x_{1},{ }_{0}^{c} D_{t}^{\beta} x_{2},{ }_{0}^{c} D_{t}^{\beta} x_{3}\right)\end{array}\right]$ 
$x_{3}(t)=\left[\begin{array}{llll}1 & 0 & 0 & 0\end{array}\right]\left[\begin{array}{c}x_{1}(t) \\ x_{2}(t) \\ x_{1 \rho}(t) \\ x_{2 \rho}(t)\end{array}\right]+f_{3}\left(x_{1}, x_{2}, x_{3}\right) \mathrm{g}_{3}\left({ }_{0}^{c} D_{t}^{\beta} x_{1},{ }_{0}^{c} D_{t}^{\beta} x_{2},{ }_{0}^{c} D_{t}^{\beta} x_{3}\right)$

$$
\begin{aligned}
& {\left[\begin{array}{cccc}
\tilde{a}_{11} & \tilde{a}_{12} & \tilde{b}_{11} K_{\rho} & \tilde{b}_{12} K_{\rho} \\
\tilde{a}_{21} & \tilde{a}_{22} & \tilde{b}_{2,1} K_{\rho} & \tilde{b}_{2,2} K_{\rho} \\
\tilde{a}_{31}\left(\tilde{b}_{11 \rho}+\tilde{b}_{12 \rho}\right) & \tilde{a}_{32}\left(\tilde{b}_{11 \rho}+\tilde{b}_{12 \rho}\right) & \sum_{j=1}^{2} \tilde{b}_{1 j \rho} \tilde{b}_{31} K_{\rho}+\tilde{a}_{11 \rho} & \sum_{j=1}^{2} \tilde{b}_{1 j \rho} \tilde{b}_{32} K_{\rho}+\tilde{a}_{12 \rho} \\
\tilde{a}_{31}\left(\tilde{b}_{21 \rho}+\tilde{b}_{22 \rho}\right) & \tilde{a}_{32}\left(\tilde{b}_{21 \rho}+\tilde{b}_{22 \rho}\right) & \sum_{j=1}^{2} \tilde{b}_{2 j \rho} \tilde{b}_{31} K_{\rho}+\tilde{a}_{21 \rho} & \sum_{j=1}^{2} \tilde{b}_{2 j \rho} \tilde{b}_{32} K_{\rho}+\tilde{a}_{22 \rho}
\end{array}\right]=} \\
& {\left[\begin{array}{llll}
2 & 0 & 0 & 0 \\
0 & 2 & 0 & 0 \\
0 & 0 & 2 & 0 \\
0 & 0 & 0 & 2
\end{array}\right]} \\
& {\left[\begin{array}{c}
f_{1}\left(x_{1}, x_{2}, x_{3}\right) g_{1}\left({ }_{0}^{c} D_{t}^{\beta} x_{1},{ }_{0}^{c} D_{t}^{\beta} x_{2},{ }_{0}^{c} D_{t}^{\beta} x_{3}\right) \\
f_{2}\left(x_{1}, x_{2}, x_{3}\right) g_{2}\left({ }_{0}^{c} D_{t}^{\beta} x_{1},{ }_{0}^{c} D_{t}^{\beta} x_{2},{ }_{0}^{c} D_{t}^{\beta} x_{3}\right) \\
\tilde{b}_{12 \rho} f_{3}\left(x_{1}, x_{2}, x_{3}\right) g_{3}\left({ }_{0}^{c} D_{t}^{\beta} x_{1},{ }_{0}^{c} D_{t}^{\beta} x_{2},{ }_{0}^{c} D_{t}^{\beta} x_{3}\right) \\
\tilde{b}_{22 \rho} f_{3}\left(x_{1}, x_{2}, x_{3}\right) g_{3}\left({ }_{0}^{c} D_{t}^{\beta} x_{1},{ }_{0}^{c} D_{t}^{\beta} x_{2},{ }_{0}^{c} D_{t}^{\beta} x_{3}\right)
\end{array}\right]=\left[\begin{array}{c}
x_{1} x_{2} K_{g_{1}}(t) \mu_{g_{1}}\left(\left\|{ }_{0}^{c} D_{t}^{\alpha} x\right\|\right) \\
x_{1} x_{3} K_{g_{2}}(t) \mu_{g_{2}}\left(\left\|{ }_{0}^{c} D_{t}^{\alpha} x\right\|\right) \\
\tilde{b}_{12 \rho} x_{2} x_{3} K_{g_{3}}(t) \mu_{g_{3}}\left(\left\|{ }_{0}^{c} D_{t}^{\alpha} x\right\|\right) \\
\tilde{b}_{22 \rho} x_{2} x_{3} K_{g_{3}}(t) \mu_{g_{3}}\left(\left\|{ }_{0}^{c} D_{t}^{\alpha} x\right\|\right)
\end{array}\right]}
\end{aligned}
$$

To compute condition (1):

Since $A=\left[\begin{array}{llll}2 & 0 & 0 & 0 \\ 0 & 2 & 0 & 0 \\ 0 & 0 & 2 & 0 \\ 0 & 0 & 0 & 2\end{array}\right]$ and the eig $(A)$ has values such as $\lambda_{1,2,3}=-2$ then

$\operatorname{Re}(\operatorname{eig}(A))<0$ and $\omega=-\max \operatorname{Re}(\operatorname{eig}(A))=2>\Gamma(\alpha)=1.772$.

\section{To compute condition (2):}

$$
\begin{gathered}
\lim _{\|\bar{x}\| \rightarrow 0} \frac{\left\|f_{1}\left(x_{1}, x_{2}, x_{3}\right) g_{1}\left({ }_{0}^{c} D_{t}^{\beta} x_{1,0}^{c} D_{t}^{\beta} x_{2},{ }_{0}^{c} D_{t}^{\beta} x_{3}\right)\right\|}{\|\bar{x}(t)\|}=\lim _{\|\bar{x}\| \rightarrow 0} \frac{\sqrt{\left(x_{1} x_{2}\right)^{2}} K_{g_{1}}(t) \mu_{g_{1}}\left(\left\|_{0}^{c} D_{t}^{\alpha} x\right\|\right)}{\sqrt{x_{1}^{2}+x_{2}^{2}+x_{3}^{2}}} \leq \\
\lim _{\|\bar{x}\| \rightarrow 0} \frac{\sqrt{x_{1}^{2} x_{2}^{2}}}{\sqrt{x_{1}^{2}}}=\lim _{\|\bar{x}\| \rightarrow 0} \sqrt{x_{2}^{2}}=\lim _{\|\bar{x}\| \rightarrow 0} \sqrt{x_{1}^{2}}=0 \\
\lim _{\|\bar{x}\| \rightarrow 0} \frac{\left\|f_{2}\left(x_{1}, x_{2}, x_{3}\right) g_{2}\left({ }_{0}^{c} D_{t}^{\beta} x_{1},{ }_{0}^{c} D_{t}^{\beta} x_{2},{ }_{0}^{c} D_{t}^{\beta} x_{3}\right)\right\|}{\|\bar{x}(t)\|}=\lim _{\|\bar{x}\| \rightarrow 0} \frac{\sqrt{\left(x_{1} x_{3}\right)^{2}} K_{g_{2}}(t) \mu_{g_{2}}\left(\left\|{ }_{0}^{c} D_{t}^{\alpha} x\right\|\right)}{\sqrt{x_{1}^{2}+x_{2}^{2}+x_{3}^{2}}} \\
\leq \lim _{\|\bar{x}\| \rightarrow 0} \frac{\sqrt{x_{1}^{2} x_{3}^{2}}}{\sqrt{x_{1}^{2}}}=\lim _{\|\bar{x}\| \rightarrow 0} \sqrt{x_{3}^{2}}=\lim _{\|\bar{x}\| \rightarrow 0} \sqrt{x_{3}^{2}}=0 \\
\lim _{\|\bar{x}\| \rightarrow 0} \frac{\left\|f_{3}\left(x_{1}, x_{2}, x_{3}\right) g_{3}\left({ }_{0}^{c} D_{t}^{\beta} x_{1},{ }_{0}^{c} D_{t}^{\beta} x_{2},{ }_{0}^{c} D_{t}^{\beta} x_{3}\right)\right\|}{\|\bar{x}(t)\|}=\lim _{\|\bar{x}\| \rightarrow 0} \frac{\sqrt{\left(x_{2} x_{3}\right)^{2}} K_{g_{3}}(t) \mu_{g_{3}}\left(\|\|_{0}^{c} D_{t}^{\alpha} x \|\right)}{\sqrt{x_{1}^{2}+x_{2}^{2}+x_{3}^{2}}} \\
\leq \lim _{\|\bar{x}\| \rightarrow 0} \frac{\sqrt{x_{2}^{2} x_{3}^{2}}}{\sqrt{x_{2}^{2}}}=\lim _{\|\bar{x}\| \rightarrow 0} \sqrt{x_{3}^{2}}=\lim _{\|\bar{x}\| \rightarrow 0} \sqrt{x_{3}^{2}}=0
\end{gathered}
$$

and $\left\|x_{3}\right\| \rightarrow 0$. Then, by theorem (2.1.1) we show that the zero solution of the system (19-20) is asymptotically stable

\subsection{Multi - Fractional Order Differential-Algebraic Control Equation}

Consider the following nonlinear multi-fractional order differential - algebraic control system:

$$
\left({ }_{0}^{c} D_{t}^{\alpha_{1}}\right) x_{1}(t)+\left({ }_{0}^{c} D_{t}^{\alpha_{2}}\right) x_{1}(t)=\sum_{i=1}^{3} a_{1 i} x_{i}(t)+\sum_{i=1}^{2} b_{1, i} u_{i}(t)+f_{1}\left(x_{1}, x_{2}, x_{3}\right) g_{1}
$$

${ }_{0}^{c} D_{t}^{\beta} x_{1},{ }_{0}^{c} D_{t}^{\beta} x_{2},{ }_{0}^{c} D_{t}^{\beta} x_{3}$ 
$\left({ }_{0}^{c} D_{t}^{\alpha_{1}}\right) x_{2}(t)+\left({ }_{0}^{c} D_{t}^{\alpha_{2}}\right) x_{2}(t)=\sum_{i=1}^{3} a_{2 i} x_{i}(t)+\sum_{i=1}^{2} b_{2, i} u_{i}(t)+f_{2}\left(x_{1}, x_{2}, x_{3}\right) g_{2}$ $\left({ }_{0}^{c} D_{t}^{\beta} x_{1}, D_{t}^{\beta} x_{2}, D_{t}^{\beta} x_{3}\right)$

$$
x_{3}(t)=\sum_{i=1}^{3} a_{3 i} x_{i}(t)+\sum_{i=1}^{2} b_{3 i} u_{i}(t)+f_{3}\left(x_{1}, x_{2}, x_{3}\right) g_{3}\left({ }_{0}^{c} D_{t}^{\beta} x_{1},{ }_{0}^{c} D_{t}^{\beta} x_{2},{ }_{0}^{c} D_{t}^{\beta} x_{3}\right)
$$

where $a_{1 i}, a_{2 i}, a_{3 i}, b_{1, i}, b_{2 i}$, and $b_{3 i}$ are constants, $x_{i} \in R$ are state vectors, $\mathrm{i}=1 . .3$

$u_{i}(t) \in R, \mathrm{i}=1,2$ are control input functions, and $f_{i}, \mathrm{~g}_{i}, \mathrm{i}=1 \ldots 3$ are varying nonlinear time values .

System (21-23) yields a linear dynamical system, as follows

$$
\begin{aligned}
& \left({ }_{0}^{c} D_{t}^{\alpha_{1}}\right) x_{1}(t)+\left({ }_{0}^{c} D_{t}^{\alpha_{2}}\right) x_{1}(t)=\sum_{i=1}^{3} a_{1 i} x_{i}(t)+\sum_{i=1}^{2} b_{1 i} u_{i}(t) \\
& \left({ }_{0}^{c} D_{t}^{\alpha_{1}}\right) x_{2}(t)+\left({ }_{0}^{c} D_{t}^{\alpha_{2}}\right) x_{2}(t)=\sum_{i=1}^{3} a_{2 i} x_{i}(t)+\sum_{i=1}^{2} b_{2 i} u_{i}(t) \\
& x_{3}(t)=\sum_{i=1}^{3} a_{3 i} x_{i}(t)+\sum_{i=1}^{2} b_{3 i} u_{i}(t)
\end{aligned}
$$

We get

$$
\begin{aligned}
& \left({ }_{0}^{c} D_{t}^{\alpha_{1}}\right) x_{1}(t)+\left({ }_{0}^{c} D_{t}^{\alpha_{2}}\right) x_{1}(t)=a_{11} x_{1}(t)+a_{12} x_{2}(t)+a_{13}\left[\frac{a_{31} x_{1}(t)}{1-a_{33}}+\frac{a_{32} x_{2}(t)}{1-a_{33}}+\frac{\sum_{i=1}^{2} b_{3 i} u_{i}(t)}{1-a_{33}}\right] \\
& +\sum_{i=1}^{2} b_{1 i} u_{i}(t) \\
& \left({ }_{0}^{c} D_{t}^{\alpha_{1}}\right) x_{2}(t)+\left({ }_{0}^{c} D_{t}^{\alpha_{2}}\right) x_{2}(t)=a_{21} x_{1}(t)+a_{22} x_{2}(t)+a_{23}\left[\frac{a_{31} x_{1}(t)}{1-a_{33}}+\frac{a_{32} x_{2}(t)}{1-a_{33}}+\frac{\sum_{i=1}^{2} b_{3 i} u_{i}(t)}{1-a_{33}}\right] \\
& +\sum_{i=1}^{2} b_{2, i} u_{i}(t) \\
& x_{3}(t)=\left[\frac{a_{31} x_{1}(t)}{1-a_{33}}+\frac{a_{32} x_{2}(t)}{1-a_{33}}+\frac{\sum_{i=1}^{2} b_{3 i} u_{i}(t)}{1-a_{33}}\right]
\end{aligned}
$$

Assume that

$\tilde{a}_{11}=a_{11}+\frac{a_{13} a_{31}}{1-a_{33}} \quad, \tilde{a}_{12}=\left[a_{12}+\frac{a_{13} a_{32}}{1-a_{33}}\right] \quad, \quad \tilde{a}_{21}=a_{21}+\frac{a_{23} a_{31}}{1-a_{33}} \quad, \tilde{a}_{22}=\left[a_{22}+\frac{a_{23} a_{32}}{1-a_{33}}\right], \tilde{a}_{31}=$ $\frac{a_{31}}{1-a_{33}}, \tilde{a}_{32}=\frac{a_{32}}{1-a_{33}}, \tilde{b}_{11}=\frac{b_{3,1}}{1-a_{33}}+b_{11}, \tilde{b}_{12}=\frac{b_{3,2}}{1-a_{33}}+b_{12}, \tilde{b}_{21}=\frac{a_{2,3} b_{3,1}}{1-a_{33}}+b_{21}, \tilde{b}_{21}=\frac{a_{2,3} b_{3,2}}{1-a_{33}}+b_{21}$ , $\tilde{b}_{31}=\frac{b_{3,1}}{1-a_{33}}, \tilde{b}_{32}=\frac{b_{3,2}}{1-a_{33}}$

Therefore

$$
\begin{aligned}
& \left({ }_{0}^{c} D_{t}^{\alpha_{1}}\right) x_{1}(t)+\left({ }_{0}^{c} D_{t}^{\alpha_{2}}\right) x_{1}(t)=\tilde{a}_{11} x_{1}(t)+\tilde{a}_{12} x_{2}(t)++\sum_{i=1}^{2} \tilde{b}_{1 i} u_{i}(t) \\
& \left({ }_{0}^{c} D_{t}^{\alpha_{1}}\right) x_{1}(t)+\left({ }_{0}^{c} D_{t}^{\alpha_{2}}\right) x_{1}(t)=\tilde{a}_{21} x_{1}(t)+\tilde{a}_{22} x_{2}(t)++\sum_{i=1}^{2} \tilde{b}_{2 i} u_{i}(t)
\end{aligned}
$$$$
x_{3}(t)=\tilde{a}_{31} x_{1}(t)+\tilde{a}_{32} x_{2}(t)+\sum_{i=1}^{2} \tilde{b}_{3 i} u_{i}(t) \text {. }
$$

Now, consider the following related linear feedback control system

$$
\begin{aligned}
& \left({ }_{0}^{c} D_{t}^{\alpha_{1}}\right) x_{1, \rho}(t)+\left({ }_{0}^{c} D_{t}^{\alpha_{2}}\right) x_{1 \rho}(t)=\tilde{a}_{11 \rho} x_{1 \rho}(t)+\tilde{a}_{12 \rho} x_{2 \rho}(t)++\tilde{b}_{11 \rho} x_{3}(t)+\tilde{b}_{12 \rho} x_{3}(t) \\
& \left({ }_{0}^{c} D_{t}^{\alpha_{1}}\right) x_{2, \rho}(t)+\left({ }_{0}^{c} D_{t}^{\alpha_{2}}\right) x_{2 \rho}(t)=\tilde{a}_{21 \rho} x_{1 \rho}(t)+\tilde{a}_{22 \rho} x_{2 \rho}(t)++\tilde{b}_{21 \rho} x_{3}(t)+\tilde{b}_{22 \rho} x_{3}(t) \\
& u_{1}(t)=K_{\rho} x_{1 \rho}(t), u_{2}(t)=K_{\rho} x_{2 \rho}(t)
\end{aligned}
$$

where $x_{1 \rho}, x_{2 \rho} \in R$, and $\tilde{a}_{11 \rho}, \tilde{a}_{12 \rho}, \tilde{a}_{21 \rho}, \tilde{a}_{22 \rho}$, and $K_{\rho}$ are constants.

We have that

$$
\begin{aligned}
& {\left[\begin{array}{c}
\left({ }_{0}^{c} D_{t}^{\alpha_{1}}\right) x_{1}(t)+\left({ }_{0}^{c} D_{t}^{\alpha_{2}}\right) x_{1}(t) \\
\left({ }_{0}^{c} D_{t}^{\alpha_{1}}\right) x_{2}(t)+\left({ }_{0}^{c} D_{t}^{\alpha_{2}}\right) x_{2}(t) \\
\left({ }_{0}^{c} D_{t}^{\alpha_{1}}\right) x_{1, \rho}(t)+\left({ }_{0}^{c} D_{t}^{\alpha_{2}}\right) x_{1, \rho}(t) \\
\left({ }_{0}^{c} D_{t}^{\alpha_{1}}\right) x_{2, \rho}(t)+\left({ }_{0}^{c} D_{t}^{\alpha_{2}}\right) x_{2, \rho}(t)
\end{array}\right]}
\end{aligned}
$$

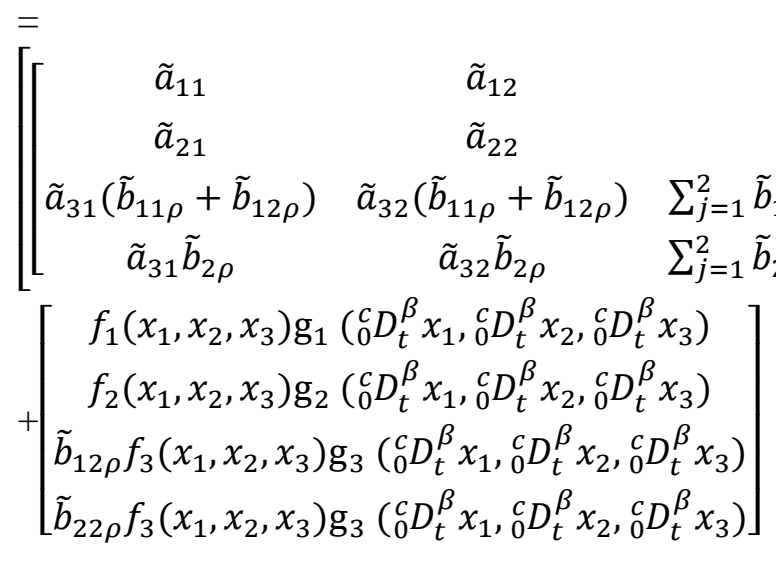$$
\left.\begin{array}{c}
\tilde{b}_{12} K_{\rho} \\
\tilde{b}_{22} K_{\rho} \\
\sum_{j=1}^{2} \tilde{b}_{1 j \rho} \tilde{b}_{32} K_{\rho}+\tilde{a}_{12 \rho} \\
\sum_{j=1}^{2} \tilde{b}_{2 j \rho} \tilde{b}_{32} K_{\rho}+\tilde{a}_{22 \rho}
\end{array}\right]\left[\begin{array}{c}
x_{1}(t) \\
x_{2}(t) \\
x_{1 \rho}(t) \\
x_{2 \rho}(t)
\end{array}\right]
$$ 
$x_{3}(t)=\left[\begin{array}{llll}\tilde{a}_{31} & \tilde{a}_{32} & \tilde{b}_{31} K_{\rho} & \tilde{b}_{32} K_{\rho}\end{array}\right]\left[\begin{array}{c}x_{1}(t) \\ x_{2}(t) \\ x_{1 \rho}(t) \\ x_{2 \rho}(t)\end{array}\right]+f_{3}\left(x_{1}, x_{2}, x_{3}\right) \mathrm{g}_{3}\left(\mathrm{t},{ }_{0}^{c} D_{t}^{\beta} x_{1},{ }_{0}^{c} D_{t}^{\beta} x_{2},{ }_{0}^{c} D_{t}^{\beta} x_{3}\right)$

Then

$$
\begin{gathered}
\left({ }_{0}^{c} D_{t}^{\alpha_{1}}\right) x(t)+\left({ }_{0}^{c} D_{t}^{\alpha_{2}}\right) x(t)=A x+F(x) G\left(\mathrm{t},{ }_{0}^{c} D_{t}^{\beta} x\right) \\
x_{3}(t)=B x+f_{3}(x) g_{3}\left(\mathrm{t},{ }_{0}^{c} D_{t}^{\beta} x\right), x=\left(x_{1}, x_{2}, x_{3}\right)
\end{gathered}
$$

where

$A=\left[\begin{array}{cccc}\tilde{a}_{11} & \tilde{a}_{12} & \tilde{b}_{11} K_{\rho} & \tilde{b}_{12} K_{\rho} \\ \tilde{a}_{21} & \tilde{a}_{22} & \tilde{b}_{2,1} K_{\rho} & \tilde{b}_{2,2} K_{\rho} \\ \tilde{a}_{31}\left(\tilde{b}_{11 \rho}+\tilde{b}_{12 \rho}\right) & \tilde{a}_{32}\left(\tilde{b}_{11 \rho}+\tilde{b}_{12 \rho}\right) & \sum_{j=1}^{2} \tilde{b}_{1 j \rho} \tilde{b}_{31} K_{\rho}+\tilde{a}_{11 \rho} & \sum_{j=1}^{2} \tilde{b}_{1 j \rho} \tilde{b}_{32} K_{\rho}+\tilde{a}_{12 \rho} \\ \tilde{a}_{31}\left(\tilde{b}_{21 \rho}+\tilde{b}_{22 \rho}\right) & \tilde{a}_{32}\left(\tilde{b}_{21 \rho}+\tilde{b}_{22 \rho}\right) & \sum_{j=1}^{2} \tilde{b}_{2 j \rho} \tilde{b}_{31} K_{\rho}+\tilde{a}_{21 \rho} & \sum_{j=1}^{2} \tilde{b}_{2 j \rho} \tilde{b}_{32} K_{\rho}+\tilde{a}_{22 \rho}\end{array}\right]$

$B=\left[\begin{array}{llll}\tilde{a}_{31} & \tilde{a}_{32} & \tilde{b}_{31} K_{\rho} & \tilde{b}_{32} K_{\rho}\end{array}\right], F(x) G\left({ }_{0}^{c} D_{t}^{\beta} x\right)=\left[\begin{array}{c}f_{1}\left(x_{1}, x_{2}, x_{3}\right) \mathrm{g}_{1}\left({ }_{0}^{c} D_{t}^{\beta} x_{1},{ }_{0}^{c} D_{t}^{\beta} x_{2},{ }_{0}^{c} D_{t}^{\beta} x_{3}\right) \\ f_{2}\left(x_{1}, x_{2}, x_{3}\right) \mathrm{g}_{2}\left({ }_{0}^{c} D_{t}^{\beta} x_{1},{ }_{0}^{c} D_{t}^{\beta} x_{2},{ }_{0}^{c} D_{t}^{\beta} x_{3}\right) \\ \tilde{b}_{12 \rho} f_{3}\left(x_{1}, x_{2}, x_{3}\right) \mathrm{g}_{3}\left({ }_{0}^{c} D_{t}^{\beta} x_{1},{ }_{0}^{c} D_{t}^{\beta} x_{2},{ }_{0}^{c} D_{t}^{\beta} x_{3}\right) \\ \tilde{b}_{22 \rho} f_{3}\left(x_{1}, x_{2}, x_{3}\right) g_{3}\left({ }_{0}^{c} D_{t}^{\beta} x_{1},{ }_{0}^{c} D_{t}^{\beta} x_{2},{ }_{0}^{c} D_{t}^{\beta} x_{3}\right)\end{array}\right]$

$f_{3}(x) g_{3}\left({ }_{0}^{c} D_{t}^{\alpha} x\right)=f_{3}\left(x_{1}, x_{2}, x_{3}\right) g_{3}\left({ }_{0}^{c} D_{t}^{\beta} x_{1},{ }_{0}^{c} D_{t}^{\beta} x_{2},{ }_{0}^{c} D_{t}^{\beta} x_{3}\right)$.

\subsubsection{The stable equivalent system}

\section{Theorem (2.2.1), [11]}

Consider the following system of fractional differential equations

$$
\left.\begin{array}{c}
{ }_{0}^{c} D_{t}^{\alpha_{1}} x_{1_{1}}(t)=f_{1}(\hat{x}, t) \\
{ }_{0}^{c} D_{t}^{\alpha_{2}} x_{2_{1}}(t)=f_{2}(\hat{x}, t) \\
\vdots \\
{ }_{0}^{c} D_{t}^{\alpha_{2}} x_{n_{1}}(t)=f_{n}(\hat{x}, t)
\end{array}\right\}
$$

$$
\begin{aligned}
& x_{i_{1}}^{(k)}(0)=x_{i_{0}}^{(k)} \quad, \quad \mathrm{i}=1, \quad 2, \ldots, \quad \mathrm{n} ; \quad \mathrm{k}=0,1, . ., m_{i}-1, \quad t \geq 0, \\
& (29) \\
& \hat{x}=\left[x_{1_{1}}(t), x_{2_{1}}(t), \ldots, x_{n_{1}}(t)\right]^{T}, f_{i} \in R,
\end{aligned}
$$

Where $f_{i}, i=1,2, \ldots, \mathrm{n}$ are continuous functions , the fractional orders defend by $m_{i}-1<\alpha_{i}<m_{i} \in$ $Z_{+}, \mathrm{i}=1,2, \ldots$, also $\mathrm{n}$ means the $\mathrm{N}$ - dimensional system of the following fractional differential equations 


$$
\begin{aligned}
& { }_{0}^{c} D_{t}^{\sigma} x_{1_{1}}(t)=x_{1_{2}}(t) \\
& { }_{0}^{c} D_{t}^{\sigma} x_{1_{M}}(t)=x_{1_{M+1}}(t), \\
& { }_{0}^{c} D_{t}^{\sigma} x_{1_{\left(m_{1}-1\right) M}}(t) \stackrel{\vdots}{=} x_{1_{\left(m_{1}-1\right) M+1}}(t) \text {, } \\
& { }_{0}^{c} D_{t}^{\sigma} x_{1_{\alpha_{1} M}}(t)=f_{1}(\hat{x}, t), \\
& { }_{0}^{c} D_{0, t}^{\sigma} x_{2_{1}}(t)=x_{2_{2}}(t) \text {, } \\
& { }_{0}^{c} D_{t}^{\sigma} x_{2_{M}}(t)=x_{2_{M+1}}(t), \\
& \begin{array}{c}
{ }_{0}^{c} D_{t}^{\sigma} x_{2_{\left(m_{2}-1\right) M}(t)} \stackrel{\vdots}{=} x_{2_{\left(m_{2}-1\right) M+1}}(t) \\
\vdots \\
{ }_{0}^{c} D_{0, t}^{\sigma} x_{2_{\alpha_{2} M}}(t)=f_{2}(\hat{x}, t),
\end{array} \\
& { }_{0}^{c} D_{0, t}^{\sigma} x_{n_{1}}(t)=x_{n_{2}}(t), \\
& { }_{0}^{c} D_{0, t}^{\sigma} x_{n_{M}}(t)=x_{n_{M+1}}(t) \\
& \begin{array}{c}
{ }_{0}^{c} D_{t}^{\sigma} x_{n_{\left(m_{n}-1\right) M}}(t)=x_{n_{\left(m_{n-1}\right) M+1}}(t), \\
\vdots \\
{ }_{0}^{c} D_{0, t}^{\sigma} x_{1_{\alpha_{n} M}}(t)=f_{n}(\hat{x}, t),
\end{array}
\end{aligned}
$$

where $\alpha_{i}=\frac{c_{i}}{d_{i}}$, g.c.d $\left(c_{i}, d_{i}\right)=1$, and $\mathrm{i}=1,2$. Also, $M$ is a lower common multiple of $c_{i}$ and $d_{i}$, $\sigma=\frac{1}{M}, \mathrm{~N}=\left(\alpha_{1}+\alpha_{2}\right) M$.

$$
x_{i_{j}}=\left\{\begin{array}{cc}
x_{i 0}^{(k)} & j=k M+1 ; k=0,1, \ldots, m_{i}-1, \mathrm{i}=1,2, \ldots, \mathrm{n} . \\
0 & \text { otherwise }
\end{array}\right.
$$

whenever $\left[x_{1_{1}}(t), x_{1_{2}}(t), \ldots, x_{1_{\alpha_{1} M}}(t), x_{2_{1}}(t), x_{2_{2}}(t), \ldots, x_{2_{\alpha_{2} M}}(t), x_{n_{1}}(t), x_{n_{2}}(t), \ldots, x_{n_{\alpha_{n} M}}(t)\right]^{T}$ is a solution of system (27-28) and $\left[x_{1_{1}}(t), x_{1_{2}}(t), \ldots, x_{n_{1}}(t)\right]^{T} \epsilon C^{m_{1}}[0, b] \times C^{m_{2}}[0, b] \times \ldots \times$ $C^{m_{n}}[0, b]$, solved system (28-29).

Whenever $\left[x_{1_{1}}(t), x_{1_{2}}(t), \ldots, x_{n_{1}}(t)\right]^{T} \epsilon C^{m_{1}}[0, b] \times C^{m_{2}}[0, b] \times \ldots \times C^{m_{n}}[0, b]$ is a solution to system (28-29)

$\left[x_{1_{1}}(t), x_{1_{2}}(t), \ldots, x_{1_{\alpha_{1} M}}(t), x_{2_{1}}(t), x_{2_{2}}(t), \ldots, x_{2_{\alpha_{2} M}}(t), x_{n_{1}}(t), x_{n_{2}}(t), \ldots, x_{n_{\alpha_{n} M}}(t)\right]^{T}=$ $\left[x_{1_{1}}(t),{ }_{0}^{c} D_{t}^{\sigma} x_{1_{1}}(t), \ldots,{ }_{0}^{c} D_{t}^{\left(\alpha_{1} M-1\right) \sigma} x_{1_{1}}(t), x_{2_{1}}(t),{ }_{0}^{c} D_{t}^{\sigma} x_{2_{1}}(t), \ldots,{ }_{0}^{c} D_{t}^{\left(\alpha_{1} M-1\right) \sigma} x_{2_{1}}(t), \ldots\right.$, $\left.x_{n_{1}}(t),{ }_{0}^{c} D_{t}^{\sigma} x_{n_{1}}(t), \ldots,{ }_{0}^{C} D_{t}^{\left(\alpha_{1} M-1\right) \sigma} x_{n_{1}}(t)\right]^{T}$ satisfies system (27-28).

Consider the following multi- fractional differential equation:

${ }_{0}^{c} D_{t}^{\alpha_{n}} x(t)+b_{10}{ }_{0}^{c} D_{t}^{\alpha_{n-1}} x(t)+\cdots+b_{n-1}{ }_{0}^{c} D_{t}^{\alpha_{n}} x(t)+\cdots+b_{n}=f(x(t)), t>0$ $x^{(k)}(0)=x_{0}^{(k)}, \mathrm{k}=0,1, \ldots, m_{n}-1$.

where $x(t) \in R, f: D \rightarrow R$ is a continues function $D \subseteq R$ and $b_{i}, \mathrm{i}=1, \ldots, \mathrm{n}$ are constant numbers. The order $\alpha_{i}, i=1,2, \ldots, n$ are rational numbers such that $m_{i}-1<\alpha_{i}<m_{i} \in Z_{+}, \mathrm{i}=1,2, \ldots, \mathrm{n}$, $\alpha_{n}>\alpha_{n-1}>\cdots>\alpha_{1}$, and $x(t) \in C[a, b]$ is a solution of system (27-28).

\section{Corollary (2.2.2), [11]}

Suppose that $f(x(t))$ is a real valued continuous function such that $f(x(t))=b_{0} x(t)$ and equation (32-33) has a unique solution $x(t) \in C[a, \infty]$. Then, the zero solution to equation (32-33) is asymptotically stable if $|\arg (\lambda)|>\frac{\gamma \pi}{2}$, where $\lambda$ is a solution to the characteristic equation $\operatorname{det}(\lambda I-$ A) $=0 . \gamma=\frac{1}{M}$, and 
$A=\left(\begin{array}{cccccc}0 & 1 & 0 & 0 & \ldots & 0 \\ 0 & 0 & 1 & 0 & \ldots & 0 \\ \vdots & \vdots & \vdots & \vdots & \ddots & \vdots \\ 0 & 0 & 0 & 0 & \ldots & 0 \\ a_{N 1} & a_{N 2} & a_{N 3} & a_{N 4} & \ldots & a_{N N}\end{array}\right)$, and $a_{N j}=\left\{\begin{array}{c}b_{0} \quad j=1 \\ -b_{n-i} \quad j=\alpha_{i} M+1, i=1,2, \ldots, n-1 \\ 0 \quad \text { otherwise }\end{array}\right.$

Theorem (2.2.3)

Consider the nonlinear multi-fractional order differential-algebraic feedback control system (26-27), such that it satisfies the following conditions:

1. $\left|\arg \left(\lambda_{k}\right)\right|>\frac{\gamma \pi}{2}$, where $\lambda$ is a solution to the characteristic equation $\operatorname{det}\left(\lambda_{k} I-A_{k}\right)=0$. $\gamma=\frac{1}{M}$ and $A_{k}=\left(\begin{array}{cccccc}0 & 1 & 0 & 0 & \cdots & 0 \\ 0 & 0 & 1 & 0 & \cdots & 0 \\ \vdots & \vdots & \vdots & \vdots & \ddots & \vdots \\ 0 & 0 & 0 & 0 & \cdots & 1 \\ a_{N 1} & a_{N 2} & a_{N 3} & a_{N 4} & \cdots & a_{N N}\end{array}\right), \mathrm{k}=1,2,3,4$

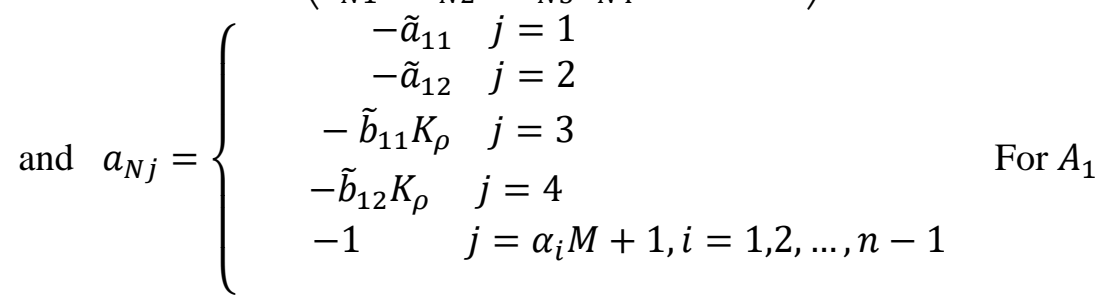

$a_{N j}=\left\{\begin{array}{cl}\tilde{a}_{21} & j=1 \\ \tilde{a}_{22} & j=2 \\ \tilde{b}_{21} K_{\rho} & j=3 \\ \tilde{b}_{22} K_{\rho} & j=4 \\ -1 & j=\alpha_{i} M+1, i=1,2, \ldots, n-1\end{array}\right.$

$a_{N j}=\left\{\begin{array}{cl}\tilde{a}_{31}\left(\tilde{b}_{1,1, \rho}+\tilde{b}_{1,2, \rho}\right) & j=1 \\ \tilde{a}_{32}\left(\tilde{b}_{1,1, \rho}+\tilde{b}_{1,2, \rho}\right) & j=2 \\ \sum_{j=1}^{2} \tilde{b}_{1, j, \rho} \tilde{b}_{3,1} K_{\rho}+\tilde{a}_{1,1, \rho} & j=3 \\ \sum_{j=1}^{2} \tilde{b}_{1, j, \rho} \tilde{b}_{3,2} K_{\rho}+\tilde{a}_{1,2, \rho} & j=4 \\ -1 \quad j=\alpha_{i} M+1, i=1,2, \ldots, n-1\end{array}\right.$

$a_{N j}=\left\{\begin{array}{c}\tilde{a}_{31} \tilde{b}_{2, \rho} \quad j=1 \\ \tilde{a}_{32} \tilde{b}_{2, \rho} \quad j=2 \\ \left.\sum_{j=1}^{2} \tilde{b}_{2, j, \rho} \tilde{b}_{3,1} K_{\rho}+\tilde{a}_{2,1, \rho}\right) \quad j=3 \\ \left(\sum_{j=1}^{2} \tilde{b}_{2, j, \rho} \tilde{b}_{3,2} K_{\rho}+\tilde{a}_{2,2, \rho}\right) \quad j=4 \\ -1 \quad j=\alpha_{i} M+1, i=1,2, \ldots, n-1\end{array}\right.$

For $A_{3}$

2. $\quad\|F(x)\|=o(\|x\|)$ as $\|x\| \rightarrow 0$.

where $F(x)=\left[\begin{array}{c}f_{1}\left(x_{1}, x_{2}, x_{3}\right) \mathrm{g}_{1}\left({ }_{0}^{c} D_{t}^{\beta} x_{1},{ }_{0}^{c} D_{t}^{\beta} x_{2},{ }_{0}^{c} D_{t}^{\beta} x_{3}\right) \\ f_{2}\left(x_{1}, x_{2}, x_{3}\right) \mathrm{g}_{2}\left({ }_{0}^{c} D_{t}^{\beta} x_{1},{ }_{0}^{c} D_{t}^{\beta} x_{2},{ }_{0}^{c} D_{t}^{\beta} x_{3}\right) \\ \tilde{b}_{2,2, \rho} f_{3}\left(x_{1}, x_{2}, x_{3}\right) \mathrm{g}_{3}\left({ }_{0}^{c} D_{t}^{\beta} x_{1},{ }_{0}^{c} D_{t}^{\beta} x_{2},{ }_{0}^{c} D_{t}^{\beta} x_{3}\right) \\ \tilde{b}_{1,2, \rho} f_{3}\left(x_{1}, x_{2}, x_{3}\right) \mathrm{g}_{3}\left({ }_{0}^{c} D_{t}^{\beta} x_{1},{ }_{0}^{c} D_{t}^{\beta} x_{2},{ }_{0}^{c} D_{t}^{\beta} x_{3}\right)\end{array}\right]$ and $K_{g_{i}}, \mathrm{i}=1, . .3$ are the nonnegative

continuous functions and $\mu_{g_{i}}$ are the continuous nondecreasing positive functions, such that $\left\|g_{i}\left(t,{ }_{0}^{c} D_{t}^{\alpha} x\right)\right\| \leq K_{g_{i}}(t) \mu_{g_{i}}\left(\left\|_{0}^{c} D_{t}^{\alpha} x\right\|\right), \mathrm{i}=1, . .3$. Then, the system is a locally asymptotically stable. 


\section{Proof}

From theorem (2.2.1), we can transform the system (24-25) which defined as folows:

$$
\begin{aligned}
& \left({ }_{0}^{c} D_{t}^{\alpha_{1}}\right) x_{1}(t)+\left({ }_{0}^{c} D_{t}^{\alpha_{2}}\right) x_{1}(t)=\tilde{a}_{11} x_{1}(t)+\tilde{a}_{12} x_{2}(t)+\tilde{b}_{11} K_{\rho} x_{1, \rho}(t)+\tilde{b}_{12} K_{\rho} x_{2, \rho}(t) \\
& \text { into a system of a single fractional order equations } \\
& { }_{0}^{c} D_{t}^{\sigma} x_{1_{1}}(t)=x_{1_{2}}(t), \\
& \vdots \\
& { }_{0}^{c} D_{t}^{\sigma} x_{1_{M}}(t)=x_{1_{M+1}}(t), \\
& \vdots \\
& { }_{0}^{c} D_{t}^{\sigma} x_{1_{\left(m_{1}-1\right) M}}(t)=x_{1_{\left(m_{1}-1\right) M+1}}(t), \\
& \vdots \\
& { }_{0}^{c} D_{t}^{\sigma} x_{1_{\alpha_{1} M}}(t)=-\tilde{a}_{11} x_{1}(t)-\tilde{a}_{12} x_{2}(t)-\tilde{b}_{11} K_{\rho} x_{1, \rho}(t)-\tilde{b}_{12} K_{\rho} x_{2, \rho}(t)
\end{aligned}
$$

such that

$$
\begin{aligned}
& A_{1}=\left[\begin{array}{cccccc}
0 & 1 & 0 & 0 & \ldots & 0 \\
0 & 0 & 1 & 0 & \ldots & 0 \\
\vdots & \vdots & \vdots & \vdots & \ddots & \vdots \\
0 & 0 & 0 & 0 & \ldots & 1 \\
a_{N 1} & a_{N 2} & a_{N 3} & a_{N 4} & \ldots & a_{N N}
\end{array}\right] \text {, and } \\
& a_{N j}=\left\{\begin{array}{cl}
-\tilde{a}_{11} & j=1 \\
-\tilde{a}_{12} & j=2 \\
-\tilde{b}_{11} K_{\rho} & j=3 \\
-\tilde{b}_{12} K_{\rho} & j=4 \\
-1 & j=\alpha_{i} M+1, i=1,2, \ldots, n-1
\end{array}\right.
\end{aligned}
$$

Now, we transfer equation

$$
\begin{aligned}
& \quad\left({ }_{0}^{c} D_{t}^{\alpha_{1}}\right) x_{1}(t)+\left({ }_{0}^{c} D_{t}^{\alpha_{2}}\right) x_{1}(t)=\tilde{a}_{21} x_{1}(t)+\tilde{a}_{22} x_{2}(t)+\tilde{b}_{21} K_{\rho} x_{1, \rho}(t)+\tilde{b}_{22} K_{\rho} x_{2, \rho}(t) \\
& \text { into } \\
& { }_{0}^{c} D_{t}^{\sigma} x_{2_{1}}(t)=x_{2_{2}}(t), \\
& \vdots \\
& { }_{0}^{c} D_{t}^{\sigma} x_{2_{M}}(t)=x_{2_{M+1}}(t), \\
& \vdots \\
& { }_{0}^{c} D_{t}^{\sigma} x_{2_{\left(m_{1}-1\right) M}}(t)=x_{2_{\left(m_{1}-1\right) M+1}}(t), \\
& \vdots \\
& { }_{0}^{c} D_{t}^{\sigma} x_{2_{\alpha_{1} M}}(t)=\tilde{a}_{21} x_{1}(t)+\tilde{a}_{22} x_{2}(t)+\tilde{b}_{21} K_{\rho} x_{1, \rho}(t)+\tilde{b}_{22} K_{\rho} x_{2, \rho}(t)
\end{aligned}
$$

such that

$$
\begin{aligned}
& A_{2}=\left[\begin{array}{cccccc}
0 & 1 & 0 & 0 & \ldots & 0 \\
0 & 0 & 1 & 0 & \ldots & 0 \\
\vdots & \vdots & \vdots & \vdots & \ddots & \vdots \\
0 & 0 & 0 & 0 & \ldots & 1 \\
a_{N 1} & a_{N 2} & a_{N 3} & a_{N 4} & \ldots & a_{N N}
\end{array}\right] \text {, and } \\
& a_{N j}=\left\{\begin{array}{cl}
\tilde{a}_{21} & j=1 \\
\tilde{a}_{22} & j=2 \\
\tilde{b}_{21} K_{\rho} & j=3 \\
\tilde{b}_{22} K_{\rho} & j=4 \\
-1 & j=\alpha_{i} M+1, i=1,2, \ldots, n-1
\end{array}\right.
\end{aligned}
$$

Now, we transfer equation

$$
\begin{aligned}
& \quad\left({ }_{0}^{c} D_{t}^{\alpha_{1}}\right) x_{1}(t)+\left({ }_{0}^{c} D_{t}^{\alpha_{2}}\right) x_{1}(t)=\tilde{a}_{31} \tilde{a}_{31}\left(\tilde{b}_{1,1, \rho}+\tilde{b}_{1,2, \rho}\right) x_{1}(t)+\tilde{a}_{32}\left(\tilde{b}_{1,1, \rho}+\tilde{b}_{1,2, \rho}\right) x_{2}(t)+ \\
& \left(\sum_{j=1}^{2} \tilde{b}_{1, j, \rho} \widetilde{b}_{3,1} K_{\rho}+\tilde{a}_{1,1, \rho}\right) x_{1, \rho}(t)+\left(\sum_{j=1}^{2} \tilde{b}_{1, j, \rho} \tilde{b}_{3,2} K_{\rho}+\tilde{a}_{1,2, \rho}\right) x_{2, \rho}(t) \\
& \text { into } \\
& { }_{0}^{c} D_{t}^{\sigma} x_{3_{1}}(t)=x_{3_{2}}(t), \\
& \vdots
\end{aligned}
$$




$$
\begin{aligned}
& { }_{0}^{c} D_{t}^{\sigma} x_{3_{M}}(t)=x_{3_{M+1}}(t), \\
& \vdots \\
& { }_{0}^{c} D_{t}^{\sigma} x_{31_{\left(m_{1}-1\right) M}}(t)=x_{3\left(m_{1}-1\right) M+1}(t), \\
& \text { : } \\
& { }_{0}^{c} D_{t}^{\sigma} x_{2_{\alpha_{1} M}}(t)=\tilde{a}_{31}\left(\tilde{b}_{1,1, \rho}+\tilde{b}_{1,2, \rho}\right) x_{1}(t)+\tilde{a}_{32}\left(\tilde{b}_{1,1, \rho}+\tilde{b}_{1,2, \rho}\right) x_{2}(t)+\left(\sum_{j=1}^{2} \tilde{b}_{1, j, \rho} \tilde{b}_{3,1} K_{\rho}+\right. \\
& \left.\tilde{a}_{1,1, \rho}\right) x_{1, \rho}(t)+\left(\sum_{j=1}^{2} \tilde{b}_{1, j, \rho} \tilde{b}_{3,2} K_{\rho}+\tilde{a}_{1,2, \rho}\right) x_{2, \rho}(t) \text {, such that } \\
& A_{3}=\left[\begin{array}{cccccc}
0 & 1 & 0 & 0 & \ldots & 0 \\
0 & 0 & 1 & 0 & \ldots & 0 \\
\vdots & \vdots & \vdots & \vdots & \ddots & \vdots \\
0 & 0 & 0 & 0 & \ldots & 1 \\
a_{N 1} & a_{N 2} & a_{N 3} & a_{N 4} & \ldots & a_{N N}
\end{array}\right] \text {, } \\
& a_{N j}=\left\{\begin{array}{cc}
\tilde{a}_{31}\left(\tilde{b}_{1,1, \rho}+\tilde{b}_{1,2, \rho}\right) & j=1 \\
\tilde{a}_{32}\left(\tilde{b}_{1,1, \rho}+\tilde{b}_{1,2, \rho}\right) & j=2 \\
\sum_{j=1}^{2} \tilde{b}_{1, j, \rho} \tilde{b}_{3,1} K_{\rho}+\tilde{a}_{1,1, \rho} & j=3 \\
\sum_{j=1}^{2} \tilde{b}_{1, j, \rho} \tilde{b}_{3,2} K_{\rho}+\tilde{a}_{1,2, \rho} & j=4 \\
-1 \quad j=\alpha_{i} M+1, i=1,2, \ldots, n-1
\end{array}\right.
\end{aligned}
$$

\section{Now, we transfer equation}

$$
\begin{aligned}
& \left({ }_{0}^{c} D_{t}^{\alpha_{1}}\right) x_{1}(t)+\left({ }_{0}^{c} D_{t}^{\alpha_{2}}\right) x_{1}(t)=\tilde{a}_{31} \tilde{b}_{2, \rho} x_{1}(t)+\tilde{a}_{32} \tilde{b}_{2, \rho} x_{2}(t)+\quad\left(\sum_{j=1}^{2} \tilde{b}_{2, j, \rho} \tilde{b}_{3,1} K_{\rho}+\right. \\
& \left.\tilde{a}_{2,1, \rho}\right) x_{1, \rho}(t)+\left(\sum_{j=1}^{2} \tilde{b}_{2, j, \rho} \tilde{b}_{3,2} K_{\rho}+\tilde{a}_{2,2, \rho}\right) x_{2, \rho}(t) \\
& \text { into } \\
& { }_{0}^{c} D_{t}^{\sigma} x_{4_{1}}(t)=x_{4_{2}}(t) \\
& \text { : } \\
& { }_{0}^{c} D_{t}^{\sigma} x_{4_{M}}(t)=x_{4_{M+1}}(t) \\
& \vdots \\
& { }_{0}^{c} D_{t}^{\sigma} x_{4_{\left(m_{1}-1\right) M}}(t)=x_{4_{\left(m_{1}-1\right) M+1}}(t) \\
& \vdots \\
& { }_{0}^{c} D_{t}^{\sigma} x_{4_{\alpha_{1} M}}(t)=\tilde{a}_{31} \tilde{b}_{2, \rho} x_{1}(t)+\tilde{a}_{32} \tilde{b}_{2, \rho} x_{2}(t)+ \\
& \left(\sum_{j=1}^{2} \tilde{b}_{2, j, \rho} \tilde{b}_{3,1} K_{\rho}+\tilde{a}_{2,1, \rho}\right) x_{1, \rho}(t)+\left(\sum_{j=1}^{2} \tilde{b}_{2, j, \rho} \tilde{b}_{3,2} K_{\rho}+\tilde{a}_{2,2, \rho}\right) x_{2, \rho}(t) \\
& \text { such that } \\
& A_{4}=\left[\begin{array}{cccccc}
0 & 1 & 0 & 0 & \ldots & 0 \\
0 & 0 & 1 & 0 & \ldots & 0 \\
\vdots & \vdots & \vdots & \vdots & \ddots & \vdots \\
0 & 0 & 0 & 0 & \ldots & 1 \\
a_{N 1} & a_{N 2} & a_{N 3} & a_{N 4} & \ldots & a_{N N}
\end{array}\right] \\
& \text { and } a_{N j}=\left\{\begin{array}{c}
\tilde{a}_{31} \tilde{b}_{2, \rho} \quad j=1 \\
\tilde{a}_{32} \tilde{b}_{2, \rho} \quad j=2 \\
\left.\sum_{j=1}^{2} \tilde{b}_{2, j, \rho} \tilde{b}_{3,1} K_{\rho}+\tilde{a}_{2,1, \rho}\right) \quad j=3 \\
\left(\sum_{j=1}^{2} \tilde{b}_{2, j, \rho} \tilde{b}_{3,2} K_{\rho}+\tilde{a}_{2,2, \rho}\right) \quad j=4 \\
-1 \quad j=\alpha_{i} M+1, i=1,2, \ldots, n-1
\end{array}\right.
\end{aligned}
$$

From condition (1), we have that $\left|\arg \left(\lambda_{k}\right)\right|>\frac{\gamma \pi}{2}$, then $A_{k}, \mathrm{k}=1,2,3,4$ are stable, and from condition (2), we have that $\| F(x(\tau)) G\left({ }_{0}^{c} D_{t}^{\beta} x(\tau)\|=\| f_{1}\left(x_{1}, x_{2}, x_{3}\right) g_{1}\left({ }_{0}^{c} D_{t}^{\beta} x_{1},{ }_{0}^{c} D_{t}^{\beta} x_{2},{ }_{0}^{c} D_{t}^{\beta} x_{3}\right) \|+\right.$

$$
\begin{aligned}
&\left\|f_{2}\left(x_{1}, x_{2}, x_{3}\right) g_{2}\left({ }_{0}^{c} D_{t}^{\beta} x_{1},{ }_{0}^{c} D_{t}^{\beta} x_{2},{ }_{0}^{c} D_{t}^{\beta} x_{3}\right)\right\| \\
&+\left\|\tilde{b}_{2,2, \rho} f_{3}\left(x_{1}, x_{2}, x_{3}\right) g_{3}\left({ }_{0}^{c} D_{t}^{\beta} x_{1},{ }_{0}^{c} D_{t}^{\beta} x_{2},{ }_{0}^{c} D_{t}^{\beta} x_{3}\right)\right\| \\
&+\left\|\tilde{b}_{12 \rho} f_{3}\left(x_{1}, x_{2}, x_{3}\right) g_{3}\left({ }_{0}^{c} D_{t}^{\beta} x_{1},{ }_{0}^{c} D_{t}^{\beta} x_{2},{ }_{0}^{c} D_{t}^{\beta} x_{3}\right)\right\|
\end{aligned}
$$


$\leq\left\|f_{1}\left(x_{1}, x_{2}, x_{3}\right)\right\| K_{g_{1}}(t) \quad \mu_{g_{1}}\left(\left\|_{0}^{c} D_{t}^{\alpha} x\right\|\right) \quad+\left\|f_{2}\left(x_{1}, x_{2}, x_{3}\right)\right\| K_{g_{2}}(t)$ $\mu_{g_{2}}\left(\left\|{ }_{0}^{c} D_{t}^{\alpha} x\right\|\right)+\left|\tilde{b}_{22 \rho}\right|\left\|f_{3}\left(x_{1}, x_{2}, x_{3}\right)\right\| K_{g_{3}}(t) \quad \mu_{g_{3}}\left(\left\|l_{0}^{c} D_{t}^{\alpha} x\right\|\right) \quad+\left|\tilde{b}_{12 \rho}\right|\left\|f_{3}\left(x_{1}, x_{2}, x_{3}\right)\right\| K_{g_{3}}(t)$ $\mu_{g_{3}}\left(\left\|{ }_{0}^{c} D_{t}^{\alpha} x\right\|\right)$.

since $\left\|F(x) G\left({ }_{0}^{c} D_{t}^{\alpha} x\right)\right\|=o(\|x\|) \quad$ as $\|x\| \rightarrow 0$, thus $\lim _{\|x\| \rightarrow 0} \frac{\left\|F(x) G\left({ }_{0}^{c} D_{t}^{\alpha} x\right)\right\|}{\|x\|}=0$, implies that $\frac{\left\|F(x) G\left({ }_{0}^{c} D_{t}^{\alpha} x\right)\right\|}{\|x\|}<\frac{1}{L_{3} L_{4}}$, where $L_{3}, L_{4}>0$. Then, we can complete the proof as in theorem (2.1.1).

Moreover, $\left\|x_{3}(t)\right\| \leq\left(B+L_{3} L_{4} K_{g_{3}}(t) \mu_{g_{3}}\left(\left\|_{0}^{c} D_{t}^{\alpha} x\right\|\right)\right)\|x\|$, hence $\left\|x_{3}(t)\right\| \rightarrow 0$.

Then, the solution of (24-25) is asymptotically stable.

\section{Example ( 2.2.4)}

Consider the following nonlinear multi-fractional order differential- algebraic with feedback control system

$$
\left[\begin{array}{l}
\left({ }_{0}^{c} D_{t}^{\alpha_{1}}\right) x_{1}(t)+\left({ }_{0}^{c} D_{t}^{\alpha_{2}}\right) x_{1}(t) \\
\left({ }_{0}^{c} D_{t}^{\alpha_{1}}\right) x_{2}(t)+\left({ }_{0}^{c} D_{t}^{\alpha_{2}}\right) x_{2}(t) \\
\left({ }_{0}^{c} D_{t}^{\alpha_{1}}\right) x_{1 \rho}(t)+\left({ }_{0}^{c} D_{t}^{\alpha_{2}}\right) x_{1 \rho}(t) \\
\left({ }_{0}^{c} D_{t}^{\alpha_{1}}\right) x_{2 \rho}(t)+\left({ }_{0}^{c} D_{t}^{\alpha_{2}}\right) x_{2 \rho}(t)
\end{array}\right]
$$

$=$

$\left.\left[\begin{array}{cccc}\tilde{a}_{11} & \tilde{a}_{12} & \tilde{b}_{11} K_{\rho} & \tilde{b}_{12} K_{\rho} \\ \tilde{a}_{21} & \tilde{a}_{22} & \tilde{b}_{2,1} K_{\rho} & \tilde{b}_{2,2} K_{\rho} \\ \tilde{a}_{31}\left(\tilde{b}_{11 \rho}+\tilde{b}_{12 \rho}\right) & \tilde{a}_{32}\left(\tilde{b}_{11 \rho}+\tilde{b}_{12 \rho}\right) & \sum_{j=1}^{2} \tilde{b}_{1 j \rho} \tilde{b}_{31} K_{\rho}+\tilde{a}_{11 \rho} & \sum_{j=1}^{2} \tilde{b}_{1 j \rho} \tilde{b}_{32} K_{\rho}+\tilde{a}_{12 \rho} \\ \tilde{a}_{31}\left(\tilde{b}_{21 \rho}+\tilde{b}_{22 \rho}\right) & \tilde{a}_{32}\left(\tilde{b}_{21 \rho}+\tilde{b}_{22 \rho}\right) & \sum_{j=1}^{2} \tilde{b}_{2 j \rho} \tilde{b}_{31} K_{\rho}+\tilde{a}_{21 \rho} & \sum_{j=1}^{2} \tilde{b}_{2 j \rho} \tilde{b}_{32} K_{\rho}+\tilde{a}_{22 \rho}\end{array}\right]\right]\left[\begin{array}{c}x_{1}(t) \\ x_{2}(t) \\ x_{1, \rho}(t) \\ x_{2, \rho}(t)\end{array}\right]$ $+\left[\begin{array}{c}f_{1}\left(x_{1}, x_{2}, x_{3}\right) \mathrm{g}_{1}\left({ }_{0}^{c} D_{t}^{\beta} x_{1},{ }_{0}^{c} D_{t}^{\beta} x_{2},{ }_{0}^{c} D_{t}^{\beta} x_{3}\right) \\ f_{2}\left(x_{1}, x_{2}, x_{3}\right) g_{2}\left({ }_{0}^{c} D_{t}^{\beta} x_{1},{ }_{0}^{c} D_{t}^{\beta} x_{2},{ }_{0}^{c} D_{t}^{\beta} x_{3}\right) \\ \tilde{b}_{12 \rho} f_{3}\left(x_{1}, x_{2}, x_{3}\right) g_{3}\left({ }_{0}^{c} D_{t}^{\beta} x_{1},{ }_{0}^{c} D_{t}^{\beta} x_{2},{ }_{0}^{c} D_{t}^{\beta} x_{3}\right) \\ \tilde{b}_{22 \rho} f_{3}\left(x_{1}, x_{2}, x_{3}\right) g_{3}\left({ }_{0}^{c} D_{t}^{\beta} x_{1},{ }_{0}^{c} D_{t}^{\beta} x_{2},{ }_{0}^{c} D_{t}^{\beta} x_{3}\right)\end{array}\right]$

$x_{3}(t)=\left[\begin{array}{llll}\tilde{a}_{31} & \tilde{a}_{32} & \tilde{b}_{31} K_{\rho} & \tilde{b}_{32} K_{\rho}\end{array}\right]\left[\begin{array}{c}x_{1}(t) \\ x_{2}(t) \\ x_{1, \rho}(t) \\ x_{2, \rho}(t)\end{array}\right]+f_{3}\left(x_{1}, x_{2}, x_{3}\right) g_{3}\left(\mathrm{t},{ }_{0}^{c} D_{t}^{\beta} x_{1},{ }_{0}^{c} D_{t}^{\beta} x_{2},{ }_{0}^{c} D_{t}^{\beta} x_{3}\right)$

where $\alpha_{1}=\frac{1}{3}, \alpha_{2}=\frac{1}{6}, \tilde{a}_{11}=\tilde{a}_{22}=\sum_{j=1}^{2} \tilde{b}_{1 j \rho} \tilde{b}_{31} K_{\rho}+\tilde{a}_{11 \rho}=\sum_{j=1}^{2} \tilde{b}_{2 j \rho} \tilde{b}_{32} K_{\rho}+\tilde{a}_{22 \rho}=-2$,

$$
\tilde{a}_{12}=\tilde{b}_{11} K_{\rho}=\tilde{b}_{12} K_{\rho}=\tilde{a}_{21}=\tilde{b}_{21} K_{\rho}=\tilde{b}_{22} K_{\rho} \tilde{a}_{31}\left(\tilde{b}_{11 \rho}+\tilde{b}_{12 \rho}\right)=\tilde{a}_{32}\left(\tilde{b}_{11 \rho}+\tilde{b}_{12 \rho}\right)=
$$

$\sum_{j=1}^{2} \tilde{b}_{1 j \rho} \tilde{b}_{32} K_{\rho}+\tilde{a}_{12 \rho}=\tilde{a}_{31} \tilde{b}_{2 \rho}=\tilde{a}_{32} \tilde{b}_{2 \rho}=\sum_{j=1}^{2} \tilde{b}_{2 j \rho} \tilde{b}_{31} K_{\rho}+\tilde{a}_{21 \rho}=0$,

$\tilde{a}_{31}=\tilde{a}_{32}=\tilde{b}_{31} K_{\rho}=0, \tilde{b}_{32} K_{\rho}=\frac{1}{2}$.

$\left[\begin{array}{c}f_{1}\left(x_{1}, x_{2}, x_{3}\right) g_{1}\left({ }_{0}^{c} D_{t}^{\beta} x_{1},{ }_{0}^{c} D_{t}^{\beta} x_{2},{ }_{0}^{c} D_{t}^{\beta} x_{3}\right) \\ f_{2}\left(x_{1}, x_{2}, x_{3}\right) g_{2}\left({ }_{0}^{c} D_{t}^{\beta} x_{1},{ }_{0}^{c} D_{t}^{\beta} x_{2},{ }_{0}^{c} D_{t}^{\beta} x_{3}\right) \\ \tilde{b}_{12 \rho} f_{3}\left(x_{1}, x_{2}, x_{3}\right) g_{3}\left({ }_{0}^{c} D_{t}^{\beta} x_{1},{ }_{0}^{c} D_{t}^{\beta} x_{2},{ }_{0}^{c} D_{t}^{\beta} x_{3}\right) \\ \tilde{b}_{22 \rho} f_{3}\left(x_{1}, x_{2}, x_{3}\right) g_{3}\left({ }_{0}^{c} D_{t}^{\beta} x_{1},{ }_{0}^{c} D_{t}^{\beta} x_{2},{ }_{0}^{c} D_{t}^{\beta} x_{3}\right)\end{array}\right]=\left[\begin{array}{c}\left.x_{1} x_{2} K_{g_{1}}(t) \mu_{g_{1}}\left(\left\|{ }_{0}^{c} D_{t}^{\alpha} x\right\|\right)\right)\|x\| \\ \left.x_{1} x_{3} K_{g_{2}}(t) \mu_{g_{2}}\left(\left\|{ }_{0}^{c} D_{t}^{\alpha} x\right\|\right)\right)\|x\| \\ \left.\tilde{b}_{12 \rho} x_{2} x_{3} K_{g_{3}}(t) \mu_{g_{3}}\left(\left\|{ }_{0}^{c} D_{t}^{\alpha} x\right\|\right)\right)\|x\| \\ \left.\tilde{b}_{22 \rho} x_{2} x_{3} K_{g_{3}}(t) \mu_{g_{3}}\left(\left\|{ }_{0}^{c} D_{t}^{\alpha} x\right\|\right)\right)\|x\|\end{array}\right]$

We have that

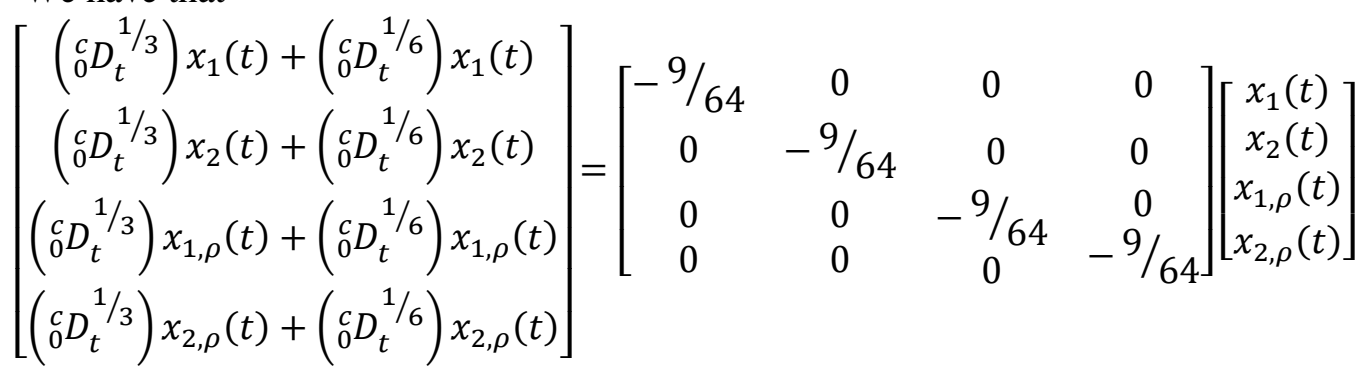


$\left.x_{3}(t)=\left[\begin{array}{llll}0 & 0 & 0 & \frac{1}{2}\end{array}\right]\left[\begin{array}{c}x_{1}(t) \\ x_{2}(t) \\ x_{1 \rho}(t) \\ x_{2 \rho}(t)\end{array}\right]+x_{2} x_{3} K_{g_{3}}(t) \mu_{g_{3}}\left(\left\|{ }_{0}^{c} D_{t}^{\alpha} x\right\|\right)\right)$

Suppose that

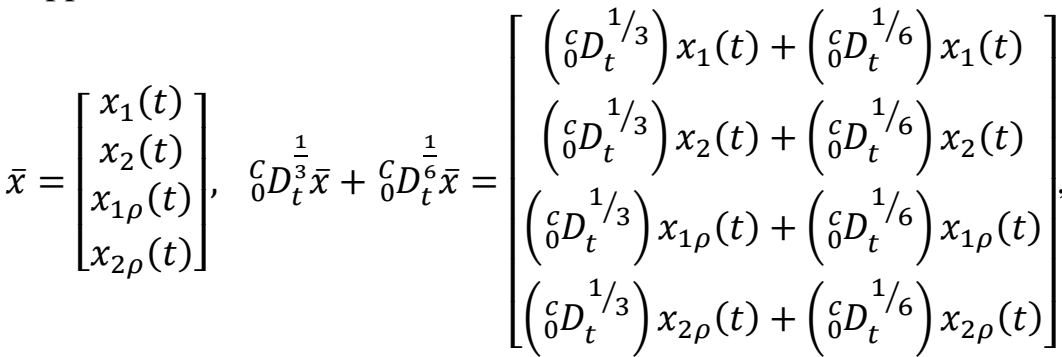

$$
\begin{aligned}
& \bar{A}=\left[\begin{array}{cccc}
-9 / 64 & 0 & 0 & 0 \\
0 & -9 / 64 & 0 & 0 \\
0 & 0 & -9 / 64 & 0 \\
0 & 0 & 0 & -9 / 64
\end{array}\right] \text {, } \\
& \bar{f}_{1}(\bar{x})=\left[\begin{array}{c}
\left.x_{1} x_{2} K_{g_{1}}(t) \mu_{g_{1}}\left(\left\|_{0}^{c} D_{t}^{\alpha} x\right\|\right)\right)\|x\| \\
\left.x_{1} x_{3} K_{g_{2}}(t) \mu_{g_{2}}\left(\left\|{ }_{0}^{c} D_{t}^{\alpha} x\right\|\right)\right)\|x\| \\
\left.\tilde{b}_{12 \rho} x_{2} x_{3} K_{g_{3}}(t) \mu_{g_{3}}\left(\left\|{ }_{0}^{c} D_{t}^{\alpha} x\right\|\right)\right)\|x\| \\
\left.\tilde{b}_{22 \rho} x_{2} x_{3} K_{g_{3}}(t) \mu_{g_{3}}\left(\left\|{ }_{0}^{c} D_{t}^{\alpha} x\right\|\right)\right)\|x\|
\end{array}\right] \text {, }
\end{aligned}
$$$$
\left.\tilde{\tilde{A}}_{2,1}=\left[\begin{array}{llll}
0 & 0 & 0 & \frac{1}{2}
\end{array}\right], \bar{f}_{2}(\bar{x})=x_{2} x_{3} K_{g_{3}}(t) \mu_{g_{3}}\left(\left\|_{0}^{c} D_{t}^{\alpha} x\right\|\right)\right)
$$$$
\left({ }_{0}^{c} D_{t}^{1 / 3}\right) x_{1}(t)+\left({ }_{0}^{c} D_{t}^{1 / 6}\right) x_{1}(t)=-9 / 64 x_{1}(t)+f_{1}\left(x_{1}, x_{2}, x_{3}\right) g_{1}\left({ }_{0}^{c} D_{t}^{\beta} x_{1},{ }_{0}^{c} D_{t}^{\beta} x_{2},{ }_{0}^{c} D_{t}^{\beta} x_{3}\right)
$$

From theorem (2.2.1), equation (36) becomes

$$
\begin{aligned}
& { }_{0}^{C} D_{t}^{\frac{1}{6}} x_{1}(t)=x_{2}(t), \\
& { }_{0}^{C} D_{t}^{\frac{1}{6}} x_{2}(t)=x_{3}(t),
\end{aligned}
$$

$$
\begin{gathered}
{ }_{0}^{C} D_{t}^{\frac{1}{6}} \bar{x}_{3}(t)=f_{1}\left(x_{1}, x_{2}, x_{3}\right) \mathrm{g}_{1}\left({ }_{0}^{c} D_{t}^{\beta} x_{1},{ }_{0}^{c} D_{t}^{\beta} x_{2},{ }_{0}^{c} D_{t}^{\beta} x_{3}\right)-x_{3}(\mathrm{t})-18 x_{1}(t) \\
{\left[\begin{array}{c}
{ }_{0} D_{t}^{\frac{1}{6}} x_{1}(t) \\
{ }_{0}^{c} D_{t}^{\frac{1}{6}} x_{2}(t)(t) \\
{ }_{0}^{C} D_{t}^{\frac{1}{6}} x_{3}(t)
\end{array}\right]=\left[\begin{array}{ccc}
0 & 1 & 0 \\
0 & 0 & 1 \\
-9 / 64 & 0 & -1
\end{array}\right]\left[\begin{array}{c}
x_{1}(t) \\
x_{2}(t) \\
x_{3}(t)
\end{array}\right]+\left[\begin{array}{c}
0 \\
0 \\
f_{1}\left(x_{1}, x_{2}, x_{3}\right) g_{1}\left({ }_{0}^{c} D_{t}^{\beta} x_{1},{ }_{0}^{c} D_{t}^{\beta} x_{2},{ }_{0}^{c} D_{t}^{\beta} x_{3}\right)
\end{array}\right]}
\end{gathered}
$$

The coefficient matrix of system (38) can be written as

$$
A_{1}=\left[\begin{array}{ccc}
0 & 1 & 0 \\
0 & 0 & 1 \\
-9 / 64 & 0 & -1
\end{array}\right] .
$$

To obtain the eigenvalues of $A_{1}$,

$$
\begin{gathered}
\operatorname{det}\left(\left[\begin{array}{ccc}
0 & \operatorname{det}\left(A_{1}-\lambda I\right)=0 \\
0 & 0 & 1 \\
-9 / 64 & 0 & -1
\end{array}\right]-\lambda\left[\begin{array}{lll}
1 & 0 & 0 \\
0 & 1 & 0 \\
0 & 0 & 1
\end{array}\right]\right)=0 \\
\operatorname{det}\left(\left[\begin{array}{ccc}
-\lambda & 1 & 0 \\
0 & -\lambda & 1 \\
-9 / 64 & 0 & -1-\lambda
\end{array}\right]\right)=0
\end{gathered}
$$




$$
\begin{gathered}
\lambda^{2}(-1-\lambda)-9 / 64=0 \\
\lambda^{3}+\lambda^{2}+9 / 64=0 \\
\Rightarrow \lambda_{1}=\lambda_{2}=\lambda_{3}=\frac{-3}{4} \\
\Rightarrow \arg \left(\lambda_{1}\right)=\frac{\pi}{6}, \quad \arg \left(\lambda_{2}\right)=\frac{-\pi}{6} \\
\Rightarrow\left|\arg \left(\lambda_{i}\right)\right|=\frac{\pi}{6}, \quad i=1,2,3 \\
\Rightarrow\left|\arg \left(\lambda_{i}\right)\right|>\frac{\gamma \pi}{2}, \quad i=1,2,3
\end{gathered}
$$

and $\frac{\gamma \pi}{2}=\frac{\pi}{12}=0.26167$

Then, from corollary (2.2.2), we have that $A_{1}$ is stable.

It is easy to

demonstrate that $\left.f_{1}\left(x_{1}, x_{2}, x_{3}\right) g_{1}\left({ }_{0}^{c} D_{t}^{\beta} x_{1},{ }_{0}^{c} D_{t}^{\beta} x_{2},{ }_{0}^{c} D_{t}^{\beta} x_{3}\right)=x_{1} x_{2} K_{g_{1}}(t) \mu_{g_{1}}\left(\left\|{ }_{0}^{c} D_{t}^{\alpha} x\right\|\right)\right)$ satisfies the following

$$
\begin{gathered}
\lim _{\|\bar{x}\| \rightarrow 0} \frac{\left\|f_{1}\left(x_{1}, x_{2}, x_{3}\right) g_{1}\left({ }_{0}^{c} D_{t}^{\beta} x_{1},{ }_{0}^{c} D_{t}^{\beta} x_{2},{ }_{0}^{c} D_{t}^{\beta} x_{3}\right)\right\|}{\|\bar{x}(t)\|}=\lim _{\|\bar{x}\| \rightarrow 0} \frac{\left.\sqrt{\left(x_{1} x_{2}\right)^{2}} K_{g_{3}}(t) \mu_{g_{3}}\left(\left\|{ }_{0}^{c} D_{t}^{\alpha} x\right\|\right)\right)}{\sqrt{\left(x_{1}\right)^{2}+\left(x_{2}\right)^{2}+\left(x_{3}\right)^{2}}} \\
\leq \lim _{\|\bar{x}\| \rightarrow 0} \frac{\sqrt{x_{1}^{2} x_{2}^{2}}}{\sqrt{x_{1}^{2}}}=\lim _{\|\bar{x}\| \rightarrow 0} \sqrt{x_{2}^{2}}=0
\end{gathered}
$$

that is $\left\|f_{1}\left(x_{1}, x_{2}, x_{3}\right) g_{1}\left({ }_{0}^{c} D_{t}^{\beta} x_{1},{ }_{0}^{c} D_{t}^{\beta} x_{2},{ }_{0}^{c} D_{t}^{\beta} x_{3}\right)\right\|=o\|x(t)\|$ as $\|x\| \rightarrow 0$.

By continuing in this way with other equations of (3), and

$\left\|x_{3}(t)\right\| \leq\left[\left\|\left[\begin{array}{llll}0 & 0 & 0 & \frac{1}{2}\end{array}\right]\right\|+L_{3} L_{4} K_{g_{3}}(t) \mu_{g_{3}}\left(\left\|_{0}^{c} D_{t}^{\alpha} x\right\|\right)\right]\|x\|$, hence $\left\|x_{3}(t)\right\| \rightarrow 0$ as $\|x\| \rightarrow 0$.

By using theorem (2.2.3), then the zero solution of equation (34-35) is asymptotically stable.

\section{Conclusions}

We studied the asymptotic stability for the proposed multi- fractional differential-algebraic control systems, involving multi control inputs, which needed to be transformed to single-fractional differential systems, using sufficient and necessary conditions.

\section{References}

1. Ahmed B. 2010. Existence Results For Fractional Differential Inclusions With Separated Boundary Conditions, Bull Korean Math. Soc. 47(4): 805-813.

2. Hasan S. Q. and Abd A. M. 2014. Robust Stabilization of Nonlinear Uncertain Descriptor Fractional Order Continuous-Time Fractional Control System, International Journal of Mathematical Archive, 5(6): 79- 89.

3. Shaheed N. Huseen S. N. 2020. On Analytical Solution of Time-Fractional Type Model of the Fisher's Equation, Iraqi Journal of Science, 61(6): 1419-1425.

4. Jabbar A. K. and Hasan S. Q. 2020. Solvability of impulsive Nonlinear Partial Differential Equation with Nonlocal Conditions, Iraqi Journal of Science, Special Issue:140-152, 2020.

5. Kaczorek T. 2018. Decentralized Stabilization of Fractional Positive descriptor Continuous-Time linear, Int J. Appl. Math. Compute. Sci., 28(1): 135-140.

6. Kaczorek T. 2018. Positivity and Stability of Descriptor Linear Systems with Interval State Matrice, Computational Problems of Electrical Engineering, 8(1).

7. Kaczorek T. 2016. Analysis of positive and stable fractional continuous-time linear systems by the use of Caputo-Fabrizio derivative by Control and Cybernetics, 45(3).

8. Men B., Zhang Q, LI X., Yang C. and Chen Y. 2006. The Stability of Linear Descriptor Systems, International Journal of information and Systems Sciences Computing and Information, 2(3): 362374. 
9. Sajewski L. 2017. Stabilization of positive descriptor fractional discrete-time linear system with two different fractional orders by decentralized controller, Bulletin of The Polish Academy of Sciences technical Sciences, $\mathbf{6 5}(5)$.

10. Mirassadi1 S. B. and Tehrani H. A. 2016. Partial Eigenvalue Assignment in Discrete-Time Descriptor Systems via Derivative State Feedback, Amirkabir International Journal of Science \& Research Modeling, Identification, Simulation and Control (AIJ-MISC), 48(2): 65-74.

11. Mirassadi S B. and Tehrani H. A. 2019. Partial eigenvalue assignment for stabilization of descriptor Fractional discrete-time linear systems, Journal of Mathematical Modeling, 7(2): 199220.

12. Guo Y. and Lin C and Wang Q. G. 2018. Stabilization for Singular Fractional-Order Systems via Static Output Feedback, IEEE Access, 6, 2018.

13. Yin C, Chen Y Q. and Zhong S. 2014. Robust stability and stabilization of uncertain fractionalorder Descriptor nonlinear system, Preprints of the 19th World Congress .The International Federation of Automatic Control Cape Town, South Africa. August 24-29, 2014.

14. Marir S. and Chadli M. 2019. Robust Admissibility and Stabilization of Uncertain Singular Fractional-Order Linear Time-Invariant Systems, IEEE/CAA Journal of Automatica Sinica, 69(3) May 2019.

15. Hasan S. Q. and Abd A. M. 2014. Robust Stabilization of Nonlinear Uncertain Descriptor Fractional Order Continuous-Time Fractional Control System, International Journal of Mathematical Archive, 5(6): 79- 89.

16. Ahmed B. 2010. Existence Results For Fractional Differential Inclusions With Separated Boundary Conditions, Bull Korean Math. Soc. 47(4): 805-813.

17. Kaczorek T. 2011. Selected Problems of Fractional System Theory, Springer-Verlag Berlin Heidelberg.

18. Lazarevic M. 2011. Stability and Stabilization of fractional order Time Delay Systems, Scientific Technical Review, 61(1).

19. Li T. and Wang Y. 2014. Stability of a Class of Fractional-Order Nonlinear Systems, Hindawi Publishing Corporation Discrete Dynamics in Nature and Society, Volume 2014, Article ID 724270,14 pages. 